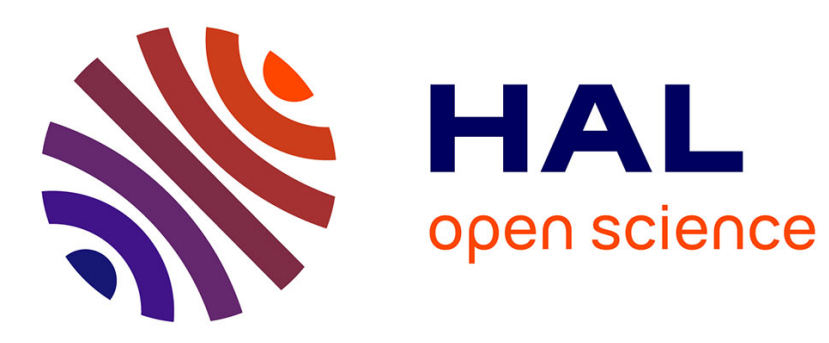

\title{
Fluid Shock Wave Generation at Solid-Material Discontinuity Surfaces in Porous Media
}

Francesco Dell'Isola, Pierre Seppecher, Angela Madeo

\section{To cite this version:}

Francesco Dell'Isola, Pierre Seppecher, Angela Madeo. Fluid Shock Wave Generation at Solid-Material Discontinuity Surfaces in Porous Media. Variational Models and Methods in Solid and Fluid Mechanics, Volume 535 of the series CISM Courses and Lectures , pp. 315-358, 2012. hal-00601626

\section{HAL Id: hal-00601626 https://hal.science/hal-00601626}

Submitted on 19 Jun 2011

HAL is a multi-disciplinary open access archive for the deposit and dissemination of scientific research documents, whether they are published or not. The documents may come from teaching and research institutions in France or abroad, or from public or private research centers.
L'archive ouverte pluridisciplinaire HAL, est destinée au dépôt et à la diffusion de documents scientifiques de niveau recherche, publiés ou non, émanant des établissements d'enseignement et de recherche français ou étrangers, des laboratoires publics ou privés. 


\title{
Fluid Shock Wave Generation at Solid-Material Discontinuity Surfaces in Porous Media
}

\author{
Francesco dell'Isola ${ }^{*}$ and Pierre Seppecher ${ }^{\dagger \ddagger}$ and Angela Madeo $+\ddagger$ \\ * Dip. DISG, Università di Roma "La Sapienza", via Eudossiana 18, 00184, \\ Rome, Italy and Laboratorio di Strutture e Materiali Intelligenti, Fondazione \\ Tullio Levi-Civita, Cisterna di Latina, Italy \\ † Inst. de Mathematique, Université de Toulon, Av. de l’Université, BP 132, \\ 83957 La Garde Cedex, France. \\ + Laboratoire de Génie Civil et Ingénierie Environnementale, Université de \\ Lyon-INSA, Bâtiment Coulomb, 69621 Villeurbanne Cedex, France \\ $\ddagger$ International Research Centre on "Mathematics \& Mechanics of Complex \\ Systems" M\& MOCS
}

\begin{abstract}
A general set of boundary conditions at the interface between dissimilar fluid-filled porous matrices is established starting from an extended Hamilton-Rayleigh principle. These conditions do include inertial effects. Once linearized, they encompass boundary conditions relative to volume Darcy-Brinkman and to surface Saffman-Beavers-Joseph-Deresiewicz dissipation effects.
\end{abstract}

\section{Introduction}

Many interesting mechanical phenomena occur in porous media when the saturating fluid flows under the action of pressure and the solid matrix is deformable. Modeling these phenomena represents an important challenge for engineering sciences.

The aim of this paper is to use the principle of virtual work -when dissipative and inertial effects cannot be neglected- for deducing a set of evolution equations and coherent boundary conditions valid at a fluid-permeable interface between dissimilar fluid-filled porous matrices. The spirit of the approach adopted here for modeling porous systems is very similar to the one used to develop models for two fluid mixtures by Gavrilyuk et al. (1997), Gavrilyuk et al. (1998), Gavrilyuk and Perepechko (1998), Gouin and Gavrilyuk (1998), Gavrilyuk and Gouin (1999), Gouin (1990). It also has some similarities with the treatment used to describe fluid saturated porous media by Dormieux and Stolz (1992), Dormieux et al. (1991) and 
Coussy and Bourbie (1984). To frame the results presented here and to compare them with those available in the literature it is necessary to detail some of the features of the model we develop. Slightly modifying the conceptual scheme used by e.g. Dormieux et al. (1991) we conceive a kinematical description which seems suitable to model porous systems which are open with respect to the fluid constituent: i.e. systems in which the fluid can freely leave or enter the porous solid matrix through which it flows. Indeed, while we still use as a basic kinematical descriptor the solid matrix macroscopic placement field $\chi_{s}$ we replace the fluid macroscopic placement field $\chi_{f}$ with a macroscopic field $\phi_{s}$ defined in the homogenized macroscopic solid reference configuration. The field $\phi_{s}$ maps any solid material particle $\mathbf{X}_{s}$ into a precise particle $\mathbf{X}_{f}$ in the fluid reference configuration: it is the fluid material particle which occupies, at the given instant, the same spatial position as $\mathbf{X}_{s}$. The adjective "macroscopic" in the previous sentences is intended to remind to the reader that the model which is used here does not attempt to describe in a detailed way how the complex geometrical structure of the pores varies in the deformation process (see infra for a discussion of this point). Obviously the placement field $\chi_{f}$ can be easily recovered as it equals $\left(\chi_{s} \circ \phi_{s}^{-1}\right)$.

The introduced kinematical description is adapted to describe the evolution of porous systems in which the solid matrix is open to fluid filtration (as it happens in consolidation problems, see e.g. Mandel (1953), Cryer (1963), Madeo (2008)). For such open systems, it is necessary to follow the placement of a solid matrix when an unknown amount of fluid, taken from a given reservoir and free to enter or leave the solid matrix, is saturating its pores and flowing through its interconnection canals.

It has to be remarked explicitly that our approach is "purely macroscopic" and "variational". The approach is purely "macroscopic" because the kinematical descriptors for the solid matrix and saturating fluid can be regarded as "averaged" placement fields obtained from corresponding "microscopic" ones (see infra). Moreover, in the scheme we use, all "microscopic" descriptors which may be relevant are assumed to be given by constitutive equations depending on the two previously introduced basic "macroscopic" placements $\chi_{s}$ and $\phi_{s}$. Here, differently to what done for instance in Dormieux and Stolz (1992), we do not attempt to deduce any "macroscopic" constitutive equations from those valid at "microscopic" level. The approach is "variational" because the evolution equations for the kinematical fields are deduced by paralleling the Hamilton-Rayleigh approach. The variational approach has been successfully adapted to continuous systems in different contexts: see e.g. Seliger and Witham (1968), Germain (1973), Houlsbya, Puzrin (2002), Sonnet et al. (2004) for (dissipative or non- 
dissipative) Cauchy continua, Bedford and Drumheller (1979) for porous media, Bedford and Drumheller (1978) for immiscible mixtures, Bedford and Drumheller (1983) for structured mixtures and in Mobbs (1982) for viscous fluids.

In this paper we construct an action functional accounting for all conservative phenomena occurring in the system and a Rayleigh dissipation function (i.e. a dissipation given in terms of a quadratic pseudo-potential). When formulating the principle of virtual work, we assume that the work done by inertial and internal conservative forces can be expressed as the first variation of the action functional and that the work done by dissipative actions can be expressed in terms of the Rayleigh dissipation function. The action-based postulation scheme is well posed as the introduced kinematical fields $\chi_{s}$ and $\phi_{s}$ are both functions defined on the solid matrix reference configuration. To be able to deal with systems in which surface solid material discontinuities are present we allow $\chi_{s}$ and $\phi_{s}$ to present gradient discontinuities concentrated on surfaces. In our analysis we generalize some results found in Batra et al. (1986).

We recover the bulk evolution equations, already available in the literature, which are valid in the regularity points of the kinematical fields. We obtain the boundary conditions valid at solid material discontinuity surfaces which are open to fluid flow. These boundary conditions may be interpreted as a "surface balance of force" and a "surface continuity of chemical potential". An Eulerian form of the first of these conditions (including inertial terms) has been obtained in Dormieux et al. (1991) where the principle of virtual work was applied to multiphase systems.

Several authors (see e.g. Ochoa-Tapia and Whitaker (1995), Jager and Mikelic (2000), Ochoa-Tapia (1995b), Hassanizadeh and Gray (1989)) formulated different boundary conditions to be used at solid material interfaces separating porous media and pure fluid. The main part of their efforts was directed to the justification and discussion of the boundary conditions originally proposed by Beavers and Joseph (1967), and Saffman (1971) for describing dissipation phenomena at the external interface of a porous systems. Some authors also focused on the deduction of such conditions by means of a micro-macro identification method: see e.g. Buridge and Keller (1981), Prat (1988), Chateau and Dormieux (1998), Marle (1982), Chandesris and Jamet (2006), Chandesris and Jamet (2007), Ochoa-Tapia and Withaker (1995) or Valdes-Paradaa et al. (2006) and references there cited. On the other hand, in Deresiewicz (1963) a set of boundary conditions valid at interfaces between dissimilar fluid-filled porous media are proposed which assure uniqueness of the solution of field equations proposed by Biot (1956).

The boundary condition stating the "surface continuity of chemical po- 
tential" obtained in the present paper include an inertial term which, to our knowledge, is not deduced in the literature. It generalizes the condition found e.g. in Baek and Srinivasa (2004) (the spirit of which is very similar to the one adopted here). Several versions of "surface balance of force" or "kinematical boundary conditions" can be found in Deresiewicz (1963), Ciezko and Kubik (1998a-b), Debergue et al. (1999), Goyeau et al. (2003), Haber and Mauri (1983), Kuznetsov (1997), Le Bars and Grae Worster (2006), Levy and Sanchez-Palencia (1975), Rajagopal and Tao (1995), Sharma (2008), Ochoa-Tapia and Whitaker (1998).

Our boundary conditions seem suitable to describe (macroscopically) the behavior of solid material interfaces open to fluid flow. They are deduced without introducing any "small perturbation" assumption, so that they seem suitable also when the assumptions of small deformations of the solid matrix and linearized Stokes fluid flow cannot be accepted. However our results are subject to all the limitations implicit in any Rayleigh-like description of dissipative phenomena based on the introduction of a pseudopotential.

The newly found boundary conditions are effectively Galilean invariant. To check this statement we revisited Reynolds Transport Theorem and Hadamard Conditions to derive some kinematical formulas implicitly used already by Gavrilyuk et al. (1997-1998). In these papers these formulas were needed to show that some evolution equations and boundary conditions assume the form of conservation laws.

We explicit warn the reader that: i) we decided to introduce an explicit notation for distinguish fields defined on the solid-reference configuration from those defined on the spatial or fluid-reference configuration, ii) we found more convenient to deduce all kinematical formulas using a space-time (Galilean) four-dimensional formalism, iii) we did separate all kinematical deductions and properties from those which are more physical in nature.

Concerning point i) we start remarking that when studying one constituent continua it is possible to proceed in presence of an abuse of notation in which fields defined in different configurations (and therefore corresponding to different mathematical functions) are denoted with the same symbol. This does not seem careful enough when multicomponent continua are considered. Indeed such an abuse of notation is, in this case, even more risky than usual, as one is dealing with models where it is necessary to introduce many different placement fields and where discontinuity surfaces for at least one of these fields may be present. In fluid-saturated porous media at least three configurations, and therefore domains of definitions for all considered fields, need to be considered. Therefore we use a notation which is more precise than the usual one, as it allows us to specify clearly for every consid- 
ered tensor field in which spatial or material domain it is defined. Should the reader be disturbed by the notation which we introduced he is invited to recover the standard one simply ignoring all the circled superscripts.

Concerning point ii) we remark that it is simpler and more convenient to consider (as done for instance by Gavrilyuk and Gouin (1999)) the four-dimensional Galilean space-time as domain for all handled kinematical fields. In this way: a) any moving bi-dimensional surfaces in the physical space becomes a fixed co-dimension one surface in four dimensional space-time, b) piecewise regular spatial fields depending on time when regarded as fields with domain in four-dimensional space-time suffer discontinuities across fixed surfaces, c) space and time differentiation, space gradients and time derivatives, deformation gradients and velocities are dealt with in a more compact and unified manner, d) Hadamard jump conditions and Reynolds transport theorems assume a very simple form, e) as a consequence, some useful -but involved- kinematical relationships are easily seen to stem from elementary differential geometric ones. Indeed, the fourdimensional Galilean space-time is the suitable setting to be used in order to deduce from some well-known results in differential geometry many properties of piecewise differentiable tensor fields. Even if it seems possible to consider weaker regularity conditions (see e.g. Savar and Tomarelli (1998)) we try to render the presentation the simplest possible still choosing the admissible kinematical fields to be general enough to describe the phenomena we have in mind.

Concerning point iii): in our deduction it was necessary to deal with some important topics in differential geometry, concerning the mathematical properties of tensor fields which can be expressed as gradients of other tensor fields. In the four-dimensional setting we have chosen, this is equivalent to study kinematical properties of multicomponent continua. Sometimes this kinematical study is presented together with topics the nature of which is more specifically mechanical, i.e. related to the postulation scheme -based on phenomenological considerations- which is assumed in a specific modeling situation. We have chosen to keep separate all kinematical considerations. The abuse of notation mentioned at point i) is even more misleading when kinematical assumptions for placement fields are mixted with the phenomenological ones characterizing either the solid or the fluid constituent behavior. The conjunction of all these confusing choices may loose the reader in an indistinct list of properties the origin of which is unclear.

Referring to de Boer (1996-2000-2005), Rajagopal and Tao (1995), Dormieux et al. (2006) for an exhaustive and clear review of the development of porous media theory we limit ourselves to recall the pioneering works of Fillunger 
(1936) (which were made available to the engineers community by Terzaghi (1943) and Biot (1941)).

The stream of research efforts which were thus originated produced several different families of mathematical models differing in the detail in which they aim to describe the reference and current configurations of solid and fluid constituents.

Indeed, the solid matrix, when displacing from its reference configuration, occupies a different spatial region which delineates a different empty pore region left to the fluid constituent. Such a region can have a very complex time-variable shape: therefore the complete description of its evolution is correspondingly very difficult. Depending on the detail which is required in such a description one can introduce a macroscopic or a microscopic model.

In the context of the theory of porous solids a "purely microscopic model" is one in which the kinematical description allows for the complete characterization of the shapes of all matrix internal pores and of fluid density and velocity at any point inside these pores. In the present paper, instead, we consider a mathematical model for the description of saturating-fluid flow in a porous matrix (having enough interconnected pores so to allow such flow) which is purely macroscopic in nature. In purely macroscopic models the "internal" shape of the porous solid matrix, i.e. the shape of its internal pores, is not described by any kinematical field and therefore the solid matrix kinematical description is limited to the introduction of a "homogenized" or "macroscopic" placement field $\chi_{s}$. This field is defined on a "homogenized" reference configuration for the solid matrix in which a solid material particle represents a cluster of pores together with that part of solid matrix which is delineating them (for a discussion of the mentioned homogenization procedure see e.g. Marle (1982), De Buhan et al. (1998a-b), Hornung (1997)). The placement of such a macroscopic particle represents the spatial region occupied by the quoted cluster of pores: clearly the Eulerian mass density related to it is related to the solid mass effectively placed in the given Eulerian volume. Thus an "apparent" solid mass density, differing from the mass density of the material constituting the solid matrix, is associated to the introduced macroscopic solid placement field. Similarly the description of the kinematics of the fluid constituent flowing through the pores, delineated by the solid matrix, is obtained in a purely macroscopic model by means of the "homogenized" placement function $\chi_{f}$ defined on a "homogenized" fluid reference configuration. The velocity and apparent mass density related to such a macroscopic placement field do not account for the variations of the "microscopic" fluid velocity and mass density fields which occur inside the pores. Recall that in the present paper we prefer to 
consider the field $\phi_{s}$ instead of $\chi_{f}$ : this is more convenient as $\phi_{s}$ is defined in the same domain as $\chi_{s}$.

One particular aspect of purely macroscopic models has been sometimes regarded as their main conceptual weakness. It concerns the physical interpretation which has to be associated to their basic kinematical descriptors, i.e. the "homogenized" placement fields: indeed a "homogenized" solid particle occupies, at a given instant, the same place as a "homogenized" solid particle. Nevertheless this circumstance is not surprising if one carefully considers the conceptual modeling assumptions underlying purely macroscopic models. The intuitive interpretation we just came to give to purely macroscopic theories for fluid saturated porous solids gains merit once grounded from a mathematical point of view by the so called "Theory of Homogenization" i.e. the mathematical theory aiming to rigorously deduce macroscopic models from microscopic ones Hornung (1997) with the references there cited, Chateau and Dormieux (1995)).

The importance of the Theory of Homogenization cannot be denied. However it is always possible and very useful to formulate "directly" a macroscopic theory without being forced to deduce it from a "purely microscopic" one. Moreover up to now very few results are available about the rigorous deduction of the macroscopic theory of Darcy flow through a deformable porous medium.

Indeed, it is always possible (and often suitable) to develop a macroscopic model independently from any microscopic one. Recall that Cauchy continuum mechanical models for one constituent bodies are formulated in a "direct" way without any reference to "atomistic" or "molecular" models and that very few practical models are rigorously justified by means of homogenization procedures. In general a mathematically coherent macroscopic model can be always formulated and supplies a useful guidance to the deduction procedure which starts from microscopic models. These procedures are often used to supply effective macroscopic constitutive equations in terms of the relevant microscopic properties of considered systems Allaire (1989-1991), for deduction of rigorous results concerning Darcy flow and to Pan and Horne (2001) Lee (2004), Kaasschieter and Frijns (2003) and references there cited for those concerning deformable matrices).

One can call "microscopic models" those intermediate models in which the macroscopic kinematical description is refined enough to describe in a more or less detailed way the shapes of the spatial regions separately occupied by solid and fluid constituents and some aspects of the motion of the material occupying these regions. The more detailed is the description of the shape of the solid porous matrix, the more "microscopic" is the formulated model. It is clear that different microscopic features of the pore 
shapes may be retained in the kinematical description : in some descriptions one could decide to account only for the ratio of volumes of the regions occupied respectively by solid and fluid constituents (thus introducing the solid volume fraction kinematical field) or for the shape of the canals interconnecting the pore (thus introducing a tortuosity tensor field) or for some geometrical features of the pores (thus introducing, for instance, the ratio between two characteristic lengths of the pore). Adding more and more kinematical descriptors one can more and more precisely approximate the purely microscopic theory. The choice of an "approximating" or "intermediate" microscopic theory results from a compromise between the need of a precise description of complex phenomena and the (computational or analytical) difficulties encountered.

We conclude remarking that the aforementioned modeling efforts respond to a strong demand from applications. Innumerable engineering problems require the design and the control of complex systems in which the flow of a fluid occur in a region partially occupied by a deformable solid matrix, the pores of which are interconnected.

Soil mechanics, geotechnical engineering and geology must supply the theoretical tools for controlling consolidation and subsidence phenomena, which are often influenced by related fluid filtration or flow phenomena (see e.g. Mandel (1953), Terzaghi (1943)) or the phenomena involved in earthquakes (see e.g. Yang (1999) or in the bradyseism and in the related micro-earthquakes in the Phlegraean Fields - Campi Flegrei region (South Italy) (see dell'Isola a et al (1998) and references there cited, Casertano et al (1976), Orsi et al. (1999)).

In biomechanics some phenomena related to the flow of fluids in a deformable porous matrix are also of interest: bone tissues are porous and several different fluids, with different properties, filtrate or flow through those pores which are interconnected. Indeed it seems now evident that bone tissue growth is regulated by a feed-back control system in which the effect of tissue deformation on fluid flow plays a central role (see e.g. Cowin (2001))

Underground engineering (e.g. when designing or maintaining underground cavities for stocking nuclear wastes or gas) also has to face relevant problems involving phenomena of fluid filtration and flow in a porous matrix coupled to cracks growth and related increase of pore volume fraction and cracks interconnection (see e.g. dell'Isola et al. (2000), dell'Isola et al. (2003) and references there quoted). 


\section{Properties of the Gradients of $C^{2}$ Vector Fields}

In this section the properties of smooth tensor fields which are the gradient of some vector field are investigated.

It is usual practice in mathematical physics, when transporting tensor fields by means of changes of variables, to use the same notation for the different tensor fields. This leads to some difficulties which are overcome when dealing with a one-constituent medium by introducing the adapted notations of material and Eulerian space-time derivatives. Such abuse of notation can be very misleading in the case of multi-constituents media where several diffeomorphisms are present. This is why we introduce a more precise notation which, although burdening, seems to be unavoidable in this context.

Notation 1. Let $\mathbb{B}_{a}$ and $\mathbb{B}_{b}$ be two regular open subdomains of $\mathbb{R}^{n}$, and let $\dddot{X}$ be an homeomorphism from $\mathbb{B}_{a}$ onto $\mathbb{B}_{b}$. Given two tensor fields $\mathbb{t}$ and $\mathbb{z}$ defined on $\mathbb{B}_{a}$ and $\mathbb{B}_{b}$ respectively, we denote

$$
\mathbb{t}^{(b)}:=\mathbb{t} \circ \boldsymbol{X}^{-1}, \quad \mathbb{z}^{\circledR}:=\mathbb{z} \circ \boldsymbol{X} .
$$

It is trivial that $\left(\mathrm{t}^{(b)}\right)^{(}=\mathrm{t}$ and $\left(\mathbf{z}^{(a)}\right)^{(b)}=\mathbf{z}$.

Notation 2. For any differentiable $k$-th order tensor field $\mathbb{t}=\mathbb{t}_{i_{1} \ldots i_{k}}$ we denote by $\mathbb{\nabla} \mathrm{t}$ its gradient and, when $k \geq 1$ and $i_{k}$ varies in $\{1,2, \ldots, n\}$, by $\mathbb{D} \mathbb{V} \mathbb{t}$ its divergence.

The components ofe these tensors are given by ${ }^{1}$

$$
\begin{array}{r}
(\mathbb{W} \mathbf{t})_{i_{1} i_{2} \ldots i_{k+1}}:=\partial_{i_{k+1}}\left(\mathbb{t}_{i_{1} i_{2} \ldots i_{k}}\right), \\
(\mathbb{D I V} \mathbb{t})_{i_{1} i_{2} \ldots i_{k-1}}:=\sum_{i_{k}=1}^{n} \partial_{i_{k}}\left(\mathbb{t}_{i_{1} i_{2} \ldots i_{k}}\right) .
\end{array}
$$

Notation 3. In this section we assume that $\mathcal{X}$ is a $C^{2}$-diffeomorphism and, in order to lighten notations, we simply denote $\mathbb{F}:=\mathbb{\nabla} \boldsymbol{X}$ and $\mathbb{J}:=\operatorname{det} \mathbb{F}$.

Using the chain rule, it is easy to recognize that $\mathbb{\nabla} \mathcal{X}^{-1}=\left(\mathbb{F}^{\text {(b) }}\right)^{-1}=\left(\mathbb{F}^{-1}\right)^{\text {(b) }}$ and so $\operatorname{det}\left(\mathbb{\nabla} \boldsymbol{X}^{-1}\right)=\left(\mathbb{J}^{(\mathbb{b})}\right)^{-1}=\left(\mathbb{J}^{-1}\right)^{\mathbb{b}}$. Moreover, if $\mathrm{t}$ and $\mathbb{z}$ are $C^{1}$ tensor

\footnotetext{
${ }^{1}$ Here and from now on the symbol $\partial_{j}$ indicate the partial derivative of a function with respect to the $j$-th component of its argument. Moreover in order to lighten notations, we adopt the Einstein summation convention on repeated indexes dropping the summation symbol.
} 
fields defined on $\mathbb{B}_{a}$ and $\mathbb{B}_{b}$ respectively: ${ }^{2}$

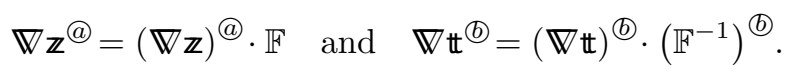

Using these notations, the well-known change of variables formula read

$$
\begin{aligned}
& \int_{\mathbb{B}_{b}} \mathbb{Z} d \mathbb{B}_{b}=\int_{\mathbb{B}_{a}} \mathbb{Z}^{@} \mathbb{J} d \mathbb{B}_{a}, \quad \text { or } \\
& \int_{\mathbb{B}_{a}} \mathbb{t} d \mathbb{B}_{a}=\int_{\mathbb{B}_{b}} \mathbb{t}^{\mathbb{(}}\left(\mathbb{J}^{-1}\right)^{\oplus} d \mathbb{B}_{b},
\end{aligned}
$$

where $d \mathbb{B}_{a}$ and $d \mathbb{B}_{b}$ denote the volume measures on $\mathbb{B}_{a}$ and $\mathbb{B}_{b}$ respectively and will be omitted in the sequel as no confusion can arise.

Next Proposition gives a transport formula for the divergence operator and states an important property for the gradient of a diffeomorphism.

Proposition 1. Let $\boldsymbol{X}$ be a $C^{2}$-diffeomorphism between the domains $\mathbb{B}_{a}$ and $\mathbb{B}_{b}$. For any differentiable tensor field $\mathbb{z}$ (of order $\geq 1$ ) defined on $\mathbb{B}_{b}$ the following equation holds

$$
\mathbb{D} \mathbb{V}\left(\mathbb{J} \mathbb{Z}^{\circledR} \cdot \mathbb{F}^{-T}\right)=\mathbb{J}(\mathbb{D} \mathbb{I} \mathbb{Z})^{\circledR} .
$$

In particular

$$
\mathbb{D} \mathbb{V}\left(\mathbb{J} \mathbb{F}^{-T}\right)=0
$$

Proof. Let us consider a differentiable scalar field $\psi$ with compact support included in $\mathbb{B}_{a}$. Owing to the regularity assumptions on $\mathcal{X}$, the corresponding scalar field $\psi^{\circledR}$ on $\mathbb{B}_{b}$ has compact support and is differentiable on $\mathbb{B}_{b}$. A simple change of variables gives

$$
\int_{\mathbb{B}_{b}} \psi^{(\mathfrak{b}} \mathbb{D} \mathbb{I V} \mathbb{Z}=\int_{\mathbb{B}_{a}} \psi(\mathbb{D} \mathbb{I} \mathbb{Z})^{\circledR} \mathbb{J} .
$$

On the other hand, using the divergence theorem and recalling that $\left.\psi^{(}\right)$has compact support

$$
\int_{\mathbb{B}_{b}} \psi^{(\oplus)} \mathbb{D} \mathbb{I V} \mathbb{Z}=-\int_{\mathbb{B}_{b}} \mathbb{z} \cdot \mathbb{\nabla} \psi^{(\bullet)}
$$

\footnotetext{
${ }^{2}$ Given two tensors $T$ and $S$ of order $k$ and $h$ the components of which are $T_{i_{1} \ldots i_{k}}$ and $S_{j_{1} \ldots j_{h}}$ the tensor $T \cdot S$ is the $(k+h)-2$ order tensor with components $(T$. $S)_{i_{1} \ldots i_{k-1} j_{2} \ldots j_{h}}=\sum_{m} T_{i_{1} \ldots i_{k-1} m} S_{m j_{2} \ldots j_{h}}$.
} 
Starting from Eq. (7), using successively formula (2) for $\psi^{(b)}$, a change of variables, the divergence theorem and the fact that $\psi$ has compact support we get the following equalities

$$
\begin{aligned}
\int_{\mathbb{B}_{b}} \psi^{(\oplus)} \mathbb{D} \mathbb{I} \mathbb{Z} & =-\int_{\mathbb{B}_{b}} \mathbb{Z} \cdot\left(\mathbb{W} \psi \cdot \mathbb{F}^{-1}\right)^{(b)}=-\int_{\mathbb{B}_{a}} \mathbb{Z}^{@} \cdot \mathbb{F}^{-T} \cdot \mathbb{\nabla} \psi \mathbb{J} \\
& =\int_{\mathbb{B}_{a}} \psi \mathbb{D} \mathbb{I V}\left(\mathbb{J} \mathbb{Z}^{@} \cdot \mathbb{F}^{-T}\right) .
\end{aligned}
$$

The comparison between Eq. (6) and the last term of Eq.(8) gives

$$
\int_{\mathbb{B}_{a}} \psi(\mathbb{D} \mathbb{V} \mathbb{Z})^{@} \mathbb{J}=\int_{\mathbb{B}_{a}} \psi \mathbb{D} \mathbb{I} \mathbb{V}\left(\mathbb{J} \mathbb{Z}^{@} \cdot \mathbb{F}^{-T}\right) .
$$

The fact that this last equality is satisfied for any $\psi$ with compact support included in $\mathbb{B}_{a}$ proves (4). It is enough to apply (4) choosing for $\mathbb{z}$ the identity tensor to get (5).

Note that the previous proposition can be applied to $\boldsymbol{X}^{-1}$ so that, for any differentiable tensor field $\mathbb{t}$ (of order $\geq 1$ ) defined on $\mathbb{B}_{a}$ one gets

$$
\begin{aligned}
& \mathbb{D} \mathbb{V}\left(\mathbb{J}^{-1} \mathbb{t} \cdot \mathbb{F}^{T}\right)^{\text {(b) }}=\left(\mathbb{J}^{-1} \mathbb{D} \mathbb{V} \mathbb{t}\right)^{(b)} \text { and } \\
& \mathbb{D} \mathbb{V}\left(\mathbb{J}^{-1} \mathbb{F}^{T}\right)^{\text {(b) }}=0 .
\end{aligned}
$$

Let $\mathbb{U}$ be a $C^{1}$ vector field defined in $\mathbb{B}_{b}$ and let $\mathbb{U} @$ be its corresponding vector field on $\mathbb{B}_{a}$. Let $\mathbb{N}_{a}$ and $\mathbb{N}_{b}$ be the outward unit normal vectors to $\partial \mathbb{B}_{a}$ and $\partial \mathbb{B}_{b}$ respectively, then

$$
\int_{\partial \mathbb{B}_{b}} \mathbb{U} \cdot \mathbb{N}_{b}=\int_{\partial \mathbb{B}_{a}} \mathbb{U}^{@} \cdot\left(\mathbb{J} \mathbb{F}^{-T} \cdot \mathbb{N}_{a}\right),
$$

Proof. Recalling Eq. (4), one gets

$$
\begin{aligned}
\int_{\partial \mathbb{B}_{b}} \mathbb{U} \cdot \mathbb{N}_{b} & =\int_{\mathbb{B}_{b}} \mathbb{D I V}(\mathbb{U})=\int_{\mathbb{B}_{a}}(\mathbb{D} \mathbb{V}(\mathbb{U}))^{\circledR} \mathbb{J} \\
& =\int_{\mathbb{B}_{a}} \mathbb{D} \mathbb{I}\left(\mathbb{J} \mathbb{U}^{@} \cdot \mathbb{F}^{-T}\right)=\int_{\partial \mathbb{B}_{a}} \mathbb{U}^{@} \cdot\left(\mathbb{J} \mathbb{F}^{-T} \cdot \mathbb{N}_{a}\right) .
\end{aligned}
$$


Note that the last corollary applied to $\boldsymbol{\mathcal { X }}^{-1}$ reads

$$
\int_{\partial \mathbb{B}_{a}} \mathbb{U}^{@} \cdot \mathbb{N}_{a}=\int_{\partial \mathbb{B}_{b}} \mathbb{U} \cdot\left(\left(\mathbb{J}^{-1} \mathbb{F}^{T}\right)^{\oplus} \cdot \mathbb{N}_{b}\right) \cdot
$$

\section{Properties of the Gradients of Piecewise $C^{1}$ Vector Fields}

In this section we define piecewise differentiable vector fields and investigate the properties of their gradients.

Let $\mathbb{S}_{a}$ be a smooth codimension-one hyper-surface in $\mathbb{B}_{a}$; this means that, at least locally, there exists a parametric representation of $\mathbb{S}_{a}$, i.e. an open subset $\Omega$ of $\mathbb{R}^{n-1}$ and a smooth embedding $\varphi \in C^{1}\left(\Omega, \mathbb{B}_{a} \subset \mathbb{R}^{n}\right)$ such that $\mathbb{S}_{a}:=\varphi(\Omega)$. By definition of an embedding, for any $\mathbf{x}=\varphi(\mathbf{s}) \in \mathbb{S}_{a}$ the vectors

$$
\mathbb{T}_{i}(\mathbf{x}):=\left.\partial_{i} \varphi\right|_{\mathbf{s}=\varphi^{-1}(\mathbf{x})}, \quad i=1,2, \ldots, n-1
$$

make a basis spanning the tangent space $\mathcal{T}_{\mathbf{x}}\left(\mathbb{S}_{a}\right)$ of $\mathbb{S}_{a}$ at $\mathbf{x}$. The orthogonal space to $\mathcal{T}_{\mathbf{x}}\left(\mathbb{S}_{a}\right)$ is one-dimensional: there exists a unique unit vector $\mathbb{N}_{a}(\mathbf{x})$ in this space which completes $\left\{\mathbb{T}_{i}(\mathbf{x})\right\}$ in a direct basis of $\mathbb{R}^{n}$. This vector $\mathbb{N}_{a}$ locally provides an orientation for $\mathbb{S}_{a}$ and we call it the normal to $\mathbb{S}_{a}$.

Notation 4. Let $\mathbb{t}$ be a tensor field defined on $\mathbb{B}_{a}$ (and consequently on $\left.\mathbb{S}_{a}\right)$. We say that $\mathrm{t}$ is differentiable on $\mathbb{S}_{a}$ if $\mathrm{t} \circ \varphi \in C^{1}(\Omega)$. The surface gradient $\mathbb{V}^{\mathbb{S}_{a}} \mathbf{t}(\mathbf{x})$ at point $\mathbf{x}$ is the linear operator which, to any tangent vector $\mathbb{T}=\sum_{i=1}^{n-1} v_{i} \mathbb{T}_{i}$, associates the derivative of $\mathbb{t}$ in the direction $\mathbb{T}$ defined by

$$
\mathbb{\nabla}^{\mathbb{S}_{a}} \mathbb{t}(\mathbf{x}) \cdot \mathbb{T}:=\sum_{i=1}^{n-1} v_{i} \partial_{i}(\mathbb{t} \circ \varphi) .
$$

Recall that, even if the basis $\mathbb{T}_{i}$ depends on the choice of the parametrization $\varphi$, the surface gradient (regarded as a linear operator) does not.

Notation 5. We say that a tensor field $\mathrm{t}$ defined on $\mathbb{B}_{a}$ is piecewise continuous (or briefly $C_{p w}^{0}$ ) if there exists a smooth codimension-one $C^{1}$ hypersurface $\mathbb{S}_{a}$ in $\mathbb{B}_{a}$ such that $\mathrm{t}$ belongs to $C^{0}\left(\mathbb{B}_{a} \backslash \mathbb{S}_{a}, \mathbb{R}^{p}\right)$ and admits continuous traces $\mathrm{t}^{+}$and $\mathrm{t}^{-}$on both sides of $\mathbb{S}_{a}$. The quantity

$$
[|\mathrm{t}(\mathrm{x})|]:=\mathbb{t}^{+}(\mathrm{x})-\mathbb{t}^{-}(\mathrm{x})
$$

is called jump of $\mathbf{t}$ through the surface $\mathbb{S}_{a}$ at point $\mathbf{x}$. Moreover, the surface $\mathbb{S}_{a}$ is said to be the singularity surface of the field $\mathrm{t}$. When $\mathrm{t}$ has vanishing 
jump across the singularity surface, we simply indicate by $\mathrm{t}$ the common value $\mathrm{t}^{+}=\mathrm{t}^{-}$.

Indeed, at least locally, the normal $\mathbb{N}_{a}$ to $\mathbb{S}_{a}$ defines the "upper" part $\mathbb{B}_{a}^{+}$ of $\mathbb{B}_{a}$ toward which the normal is pointing and the "lower" part $\mathbb{B}_{a}^{-}$of $\mathbb{B}_{a}$ in the opposite direction. Then, for any $\mathbf{x} \in \mathbb{S}_{a}$

$$
\mathbb{t}^{+}(\mathbf{x})=\lim _{\mathbf{y} \rightarrow \mathbf{x}} \mathbb{t}(\mathbf{y}), \quad \mathbf{y} \in \mathbb{B}_{a}^{+} ; \quad \mathbb{t}^{-}(\mathbf{x})=\lim _{\mathbf{y} \rightarrow \mathbf{x}} \mathbb{t}(\mathbf{y}), \quad \mathbf{y} \in \mathbb{B}_{a}^{-} .
$$

Notation 6. We say that a tensor field $\mathbb{t}$ defined on $\mathbb{B}_{a}$ is piecewise differentiable (or briefly $C_{p w}^{1}$ ) if it is continuous and if its gradient $\mathbb{\nabla} \mathbb{t}$ is $C_{p w}^{0}$.

Property 1. The well-known Hadamard property (see e.g. Kosinski (1986)) states that the jump of the gradient of a $C_{p w}^{1}$ tensor field $\mathrm{t}$ is a rank-one matrix field in the form

$$
\begin{aligned}
{[|\mathbb{\nabla} \mathrm{t}|] } & =[|\mathbb{\nabla} \mathrm{t}|] \cdot \mathbb{N}_{a} \otimes \mathbb{N}_{a}=\left((\nabla \mathrm{t})^{+} \cdot \mathbb{N}_{a}-(\nabla \mathrm{t})^{-} \cdot \mathbb{N}_{a}\right) \otimes \mathbb{N}_{a} \\
& =\left[\left|\frac{\partial \mathrm{t}}{\partial \mathbb{N}_{a}}\right|\right] \otimes \mathbb{N}_{a}
\end{aligned}
$$

In other words, for any $\mathbb{T} \in \mathcal{T}_{\mathbf{x}}\left(\mathbb{S}_{a}\right)$

$$
[|\mathbb{W} \mathbf{t}(\mathbf{x}) \cdot \mathbb{T}|]=0 \text {. }
$$

This property simply reflects the fact that $\mathrm{t}$, when restricted to $\mathbb{S}_{a}$, reduces to a differentiable field and

$$
\mathbb{W}^{\mathbb{S}_{a}} \mathrm{t} \cdot \mathbb{T}=(\mathbb{\nabla} \mathrm{t})^{+} \cdot \mathbb{T}=(\mathbb{\nabla} \mathrm{t})^{-} \cdot \mathbb{T}
$$

The following proposition states some important consequences of Property 1 .

Let $\mathbb{B}_{a}, \mathbb{B}_{b}$ be two regular open subsets of $\mathbb{R}^{n}$ respectively, and let $\mathscr{X} \in$ $C_{p w}^{1}\left(\mathbb{B}_{a}, \mathbb{B}_{b}\right)$ with singularity surface $\mathbb{S}_{a} \subset \mathbb{B}_{a}$. Assume that $\mathbb{J} \neq 0$ everywhere on $\mathbb{S}_{a}$, then

(i) For any $\mathbb{T}$ tangent to $\mathbb{S}_{a},[|\mathbb{F} \cdot \mathbb{T}|]=0$ on $\mathbb{S}_{a}$.

(ii) The surface $\mathbb{S}_{b}:=\boldsymbol{X}\left(\mathbb{S}_{a}\right)$ is a smooth codimension-one $C^{1}$ surface in $\mathbb{B}_{b}$ with tangent vectors $\left(\mathbb{F} \cdot \mathbb{T}_{i}\right)^{(b)}, i \in\{1,2, \ldots, n-1\}$.

(iii) For any normal vector field $\mathbb{M}_{b}$ to $\mathbb{S}_{b}$, the following jump condition holds on $\mathbb{S}_{a}$

$$
\left[\left|\mathbb{J}^{-1} \mathbb{F}^{T}\right|\right] \cdot \mathbb{M}_{b}^{@}=0,
$$

(iv) Moreover, the quantity $\mathbb{J}^{-1} \mathbb{F}^{T} \cdot \mathbb{M}_{b}^{@}$ which is continuous through the surface is orthogonal to $\mathbb{S}_{a}$. 
Proof. Point (i) is an immediate consequence of (12) if we recall that $\mathbb{F}$ denotes $\mathbb{\nabla} \boldsymbol{X}$. To prove point (ii) we note that, as $\boldsymbol{X}$ is $C_{p w}^{1}$, its restriction to $\mathbb{S}_{a}$ is differentiable and so is $\xi:=\boldsymbol{X} \circ \varphi$ which makes a parametrization for $\mathbb{S}_{b}:=\boldsymbol{X}\left(\mathbb{S}_{a}\right)$. Moreover, for any $\mathbf{y}=\xi(\mathbf{s}) \in \mathbb{S}_{b}$ and for any $i=1,2, \ldots, n-1$, the vectors

$$
\left.\partial_{i} \xi\right|_{\mathbf{s}=\xi^{-1}(\mathbf{y})}=\left.\left.\mathbb{F}^{ \pm}\right|_{\mathfrak{X}^{-1}(\mathbf{y})} \cdot \partial_{i} \varphi\right|_{\varphi^{-1}\left(\mathbb{X}^{-1}(\mathbf{y})\right)}=\left(\mathbb{F}^{ \pm} \cdot \mathbb{T}_{i}\right)^{(\mathbb{b}}=\left(\mathbb{F} \cdot \mathbb{T}_{i}\right)^{(b)}
$$

make a basis spanning the tangent space $\mathcal{T}_{\mathbf{y}}\left(\mathbb{S}_{b}\right)$ of $\mathbb{S}_{b}$ at $\mathbf{y}$.

To prove points (iii) and (iv) we consider, at any point $\mathbf{x}$ of $\mathbb{S}_{a}$, the three linear applications $L_{a}$ and $L_{b}^{ \pm}$respectively defined on $\mathbb{R}^{n}$ by

$$
\begin{aligned}
& L_{a}(\mathbb{U}):=\operatorname{det}\left(\mathbb{T}_{1}, \mathbb{T}_{2}, \ldots, \mathbb{T}_{n-1}, \mathbb{U}\right) \\
& L_{b}^{ \pm}(\mathbb{V}):=\operatorname{det}\left(\left(\mathbb{F}^{ \pm} \cdot \mathbb{T}_{1}\right)^{(b)},\left(\mathbb{F}^{ \pm} \cdot \mathbb{T}_{2}\right)^{(b)}, \ldots,\left(\mathbb{F}^{ \pm} \cdot \mathbb{T}_{n-1}\right)^{(b)}, \mathbb{V}\right) .
\end{aligned}
$$

Owing to point (ii) one easily gets $L_{b}^{+}(\mathbb{V})=L_{b}^{-}(\mathbb{V})$. In virtue of the Rietz theorem there exist unique vectors $\tilde{\mathbb{M}}_{a}$ and $\tilde{\mathbb{M}}_{b}$ such that

$$
L_{a}(\mathbb{U})=\tilde{\mathbb{M}}_{a} \cdot \mathbb{U}, \quad \forall \mathbb{U} \in \mathbb{R}^{n}, \quad L_{b}(\mathbb{V})=\tilde{\mathbb{M}}_{b} \cdot \mathbb{V} \quad \forall \mathbb{V} \in \mathbb{R}^{n}
$$

Hence

$$
\tilde{\mathbb{M}}_{a} \cdot \mathbb{U}=\operatorname{det}\left(\mathbb{T}_{1}, \mathbb{T}_{2}, \ldots, \mathbb{T}_{n-1}, \mathbb{U}\right)
$$

and

$$
\begin{aligned}
& \tilde{\mathbb{M}}_{b} \cdot\left(\mathbb{F}^{ \pm} \cdot \mathbb{U}\right)^{(b)} \\
& =\operatorname{det}\left(\left(\mathbb{F}^{ \pm} \cdot \mathbb{T}_{1}\right)^{(b)},\left(\mathbb{F}^{ \pm} \cdot \mathbb{T}_{2}\right)^{(b)}, \ldots,\left(\mathbb{F}^{ \pm} \cdot \mathbb{T}_{n-1}\right)^{(b)},\left(\mathbb{F}^{ \pm} \cdot \mathbb{U}\right)^{(b)}\right) \\
& =\left(\mathbb{J}^{ \pm}\right)^{(b)} \operatorname{det}\left(\left(\mathbb{T}_{1}\right)^{(b)},\left(\mathbb{T}_{2}\right)^{(b)}, \ldots,\left(\mathbb{T}_{n-1}\right)^{(b)}, \mathbb{U}^{(b)}\right) .
\end{aligned}
$$

From (18) and (19) we get

$$
\tilde{\mathbb{M}}_{b}^{@} \cdot\left(\mathbb{F}^{ \pm} \cdot \mathbb{U}\right)=\mathbb{J}^{ \pm} \operatorname{det}\left(\mathbb{T}_{1}, \mathbb{T}_{2}, \ldots, \mathbb{T}_{n-1}, \mathbb{U}\right)
$$

and so

$$
\tilde{\mathbb{M}}_{b}^{(a)} \cdot\left(\mathbb{F}^{ \pm} \cdot \mathbb{U}\right)=\mathbb{J}^{ \pm} \tilde{\mathbb{M}}_{a} \cdot \mathbb{U}
$$

Since this last identity is satisfied for any $\mathbb{U} \in \mathbb{R}^{n}$ then

$$
\tilde{\mathbb{M}}_{a}=\left(\mathbb{J}^{+}\right)^{-1}\left(\mathbb{F}^{+}\right)^{T} \cdot \tilde{\mathbb{M}}_{b}^{@}=\left(\mathbb{J}^{-}\right)^{-1}\left(\mathbb{F}^{-}\right)^{T} \cdot \tilde{\mathbb{M}}_{b}^{@},
$$

thus

$$
\left[\left|\mathbb{J}^{-1} \mathbb{F}^{T}\right|\right] \cdot \tilde{\mathbb{M}}_{b}^{@}=0
$$


From (18), (19) we also get that for any $i \in\{1,2, \ldots, n-1\}$,

$$
\tilde{\mathbb{M}}_{a} \cdot \mathbb{T}_{i}=0, \quad \text { and } \quad \tilde{\mathbb{M}}_{b} \cdot\left(\mathbb{F}^{ \pm} \cdot \mathbb{T}_{i}\right)^{(b)}=0 .
$$

Hence, $\tilde{\mathbb{M}}_{a}$ and $\tilde{\mathbb{M}}_{b}$ belong to the one dimensional orthogonal spaces to $\mathbb{S}_{a}$ and $\mathbb{S}_{b}$ respectively. As they clearly are non vanishing, Eq. (14) remains valid for any $\mathbb{M}_{b}$ normal to $\mathbb{S}_{b}$.

We call piecewise diffeomorphism a $C_{p w}^{1}$ homeomorphism $\not{X}$ from $\mathbb{B}_{a}$ onto $\mathbb{B}_{b}$ such that $\mathcal{X}^{-1} \in C_{p w}^{1}\left(\mathbb{B}_{b}, \mathbb{B}_{a}\right)$.

Note that if $\boldsymbol{X}$ is a piecewise diffeomorphism with singularity surface $\mathbb{S}_{a}$ then the previous proposition can be applied to both $\boldsymbol{X}$ and $\boldsymbol{X}^{-1}$. Thus we also have, for any $\mathbb{M}_{a} \perp \mathbb{S}_{a}$ the following jump condition on $\mathbb{S}_{b}=\boldsymbol{X}\left(\mathbb{S}_{a}\right)$

$$
\left[\left|\left(\mathbb{J} \mathbb{F}^{-T}\right)^{(6)}\right|\right] \cdot \mathbb{M}_{a}^{(\infty)}=0
$$

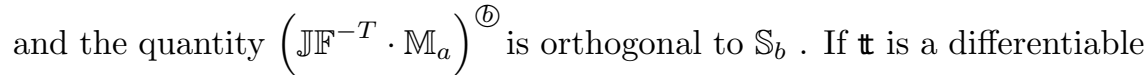
tensor field defined on $\mathbb{B}_{a}$ then its corresponding tensor field $\mathbb{t}^{(\bullet)}$ on $\mathbb{B}_{b}$ may be not differentiable on $\mathbb{S}_{b}$. However, $\mathbb{t}^{(\mathbb{D}}$ is $C_{p w}^{1}$ and on $\mathbb{S}_{b}$ we have

$$
\left(\mathbb{\nabla} \mathbb{t}^{(\mathbb{C}}\right)^{+}=\left(\mathbb{\nabla} \mathrm{t} \cdot\left(\mathbb{F}^{+}\right)^{-1}\right)^{(\mathbb{b}} ; \quad\left(\mathbb{\nabla} \mathbb{t}^{\mathbb{C}}\right)^{-}=\left(\mathbb{\nabla} \mathrm{t} \cdot\left(\mathbb{F}^{-}\right)^{-1}\right)^{\mathbb{C}} .
$$

\section{Bulk Kinematical Identities and Hadamard Conditions at Moving Boundaries}

Let $\chi: \mathbb{B}_{a}:=B_{a} \times(0, T) \rightarrow \mathbb{R}^{3}$ be the placement map of a three dimensional continuum; the fixed domain $B_{a} \subset \mathbb{R}^{3}$ is usually referred to as the reference configuration and the moving volume $B_{b}(t):=\chi\left(B_{a}, t\right)$ as the current configuration. We assume that, at any instant $t, \chi(., t)$ is a $C_{p w}^{1}$ diffeomorphism.

From now on, we denote $\mathbf{F}:=\nabla \chi$ and $\mathbf{v}:=\partial \chi / \partial t$, the usual 3D space gradient of the map $\chi$ in the domain $B_{a}$ and the usual velocity field in $B_{a}$.

The singularity surface $S_{a}(t)$ is a moving surface which can be parametrized, at least locally, by a function $\varphi:(\Omega \times(0, T)) \rightarrow B_{a}$ where $\Omega$ is an open subset of $\mathbb{R}^{2}$.

It is well known that, as only the global position of the set $S_{a}(t)$ is relevant, the physical quantities attached to $S_{a}(t)$ should not depend on the choice of its parametrization. 
From now on, we assume that $\chi$ is $C_{p w}^{1}$ on the space-time domain $\mathbb{B}_{a}$ and thus that the function $\varphi$ is $C^{1}$. Therefore it is possible to introduce, for any point $\mathbf{x}(t)=\varphi(\mathbf{s}, t) \in S_{a}(t)$, the vectors

$$
\tau_{i}(\mathbf{x}(t)):=\left.\partial_{i} \varphi\right|_{\varphi^{-1}(\mathbf{x}(t), t)}, \quad i=1,2
$$

which span a basis for the tangent plane to $S_{a}(t)$ and the unit vector $\mathbf{N}_{a}(\mathbf{x}(t))$ orthogonal to the tangent plane to $S_{a}$ which completes $\left\{\tau_{i}\right\}$ in a direct basis of $\mathbb{R}^{3}$. For any $\mathbf{x}$, we introduce the "velocity of the surface $S_{a}(t) "$ as:

$$
\mathbf{w}(\mathbf{x}(t)):=\left.\frac{\partial \varphi}{\partial t}\right|_{\varphi^{-1}(\mathbf{x}(t), t)} .
$$

It is well known, (see e.g.Kosinski (1986)), that even if this velocity depends on the choice of the parametrization $\varphi$, its normal part $c_{a}:=\mathbf{w} \cdot \mathbf{N}_{a}$, commonly called the celerity of the surface, does not. Analogously we introduce $\mathbf{N}_{b}$ and $c_{b}$ the normal and celerity of $S_{b}=\chi\left(S_{a}\right)$.

In this section we show that kinematical conditions which are usually derived in different (and sometimes intricate) ways are simple consequences of Hadamard's property. We emphasize that they are purely kinematical constraints and that neither physical assumptions nor balance principles should be used, as it is sometimes done, to derive them.

Proposition 2. For any tensor fields $f$ and $\mathbf{f}$ defined on $B_{b}$ ( $\mathbf{f}$ of order $k \geq 1$ ), we have at any regular point of $\chi$ :

$$
\begin{array}{r}
\operatorname{div}\left(J \mathbf{f}^{\circledR} \cdot \mathbf{F}^{-T}\right)=J(\operatorname{div} \mathbf{f})^{@} \text { and } \\
\frac{\partial\left(J f^{@}\right)}{\partial t}-\operatorname{div}\left(J f{ }^{@} \otimes \mathbf{F}^{-1} \cdot \mathbf{v}\right)=J\left(\frac{\partial f}{\partial t}\right)^{@},
\end{array}
$$

and in particular,

$$
\begin{array}{lc}
\operatorname{div}\left(J \mathbf{F}^{-T}\right)=0, & \frac{\partial J}{\partial t}=\operatorname{div}\left(J \mathbf{F}^{-1} \cdot \mathbf{v}\right), \\
(\operatorname{div} \mathbf{f})^{@}=\nabla \mathbf{f}^{@}: \mathbf{F}^{-1}, & \left(\frac{\partial f}{\partial t}\right)^{@}=\frac{\partial f^{@}}{\partial t}-\nabla f^{@} \cdot \mathbf{F}^{-1} \cdot \mathbf{v} .
\end{array}
$$

Moreover, at any point of the singularity surface $S_{a}$ :

$$
\begin{aligned}
& {[|\mathbf{F}|] \cdot \tau_{1}=0, \quad[|\mathbf{F}|] \cdot \tau_{2}=0, \quad[|\mathbf{F}|] \cdot c_{a} \mathbf{N}_{a}+[|\mathbf{v}|]=0,} \\
& {\left[\left|J^{-1} \mathbf{F}^{T} \cdot \mathbf{N}_{b}^{@}\right|\right]=0, \quad\left[\left|J^{-1}\left(\mathbf{v} \cdot \mathbf{N}_{b}^{@}-c_{b}^{@}\right)\right|\right]=0}
\end{aligned}
$$


and

$$
[|\mathbf{v}|] \otimes\left(J^{-1} \mathbf{F}^{T} \cdot \mathbf{N}_{b}^{@}\right)+J^{-1}\left(c_{b}^{@}-\mathbf{v} \cdot \mathbf{N}_{b}^{@}\right)[|\mathbf{F}|]=0 .
$$

Finally, the normals and celerities of $S_{a}$ and $S_{b}$ are related by

$$
\mathbf{N}_{b}=\frac{\left(J \mathbf{F}^{-T} \cdot \mathbf{N}_{a}\right)^{\text {(b) }}}{\left\|\left(J \mathbf{F}^{-T} \cdot \mathbf{N}_{a}\right)^{\text {(b) }}\right\|} \quad \text { and } \quad c_{b}=\frac{\left(J c_{a}+J\left(\mathbf{F}^{-1} \cdot \mathbf{v}\right) \cdot \mathbf{N}_{a}\right)^{(b)}}{\left\|\left(J \mathbf{F}^{-T} \cdot \mathbf{N}_{a}\right)^{\text {(b) }}\right\|} \text {. }
$$

Notice that considering $\boldsymbol{X}^{-1}$ instead of $\boldsymbol{X}$ gives equations symmetrical to $(24)-(30)$

Proof. To prove this proposition, let us extend $\chi$ in order to consider it as a fourth-dimensional piecewise diffeomorphism. We consider the $C_{p w^{-}}^{1}$ diffeomorphism $\mathcal{X}$ defined on $\mathbb{B}_{a}:=B_{a} \times(0, T)$ by $\mathcal{X}(a, t):=(\chi(a, t), t)$ and let us denote by $\mathbb{B}_{b}$ its image. The map $\boldsymbol{X}$ resumes all needed information about the motion of the considered continuum. In particular, $\mathbb{B}_{b}:=\cup_{t \in(0, T)} B_{b}(t) \times\{t\}$. The singularity surface $\mathbb{S}_{a}$ of $\boldsymbol{X}$ is related to the motion of the singularity surface $S_{a}(t)$ by $\mathbb{S}_{a}:=\cup_{t \in(0, T)} S_{a}(t) \times\{t\}$.

Notation 7. If $\mathrm{ff}$ is a tensor field of order $k \geq 1$ the components of which are $\mathbf{f}_{i_{1}, i_{2}, \ldots, i_{k}}$, with $i_{k} \in\{1,2,3,4\}$, we decompose $\mathbf{f f}$ writing $\mathbf{f f}=(\mathbf{f}, f)$. Here $\mathbf{f}$ and $f$ are the tensors of order $k$ and $k-1$ defined by

$$
\mathbf{f}_{i_{1} i_{2} \ldots i_{k-1} j}=\mathbf{f f}_{i_{1} i_{2} \ldots i_{k-1} j}, \quad j=1,2,3, \quad f_{i_{1}, i_{2}, \ldots, i_{k-1}}=\mathbb{f}_{i_{1} i_{2} \ldots i_{k-1}} 4 .
$$

Using this decomposition for $\mathrm{ff}$ and $\mathbb{U}$ we write

$$
\mathbf{f} \cdot \mathbb{U}=\mathbf{f} \cdot \mathbf{u}+f u .
$$

Notation 8. The $4 D$ space-time gradient and divergence of a tensor $\mathrm{ff}$ defined in the space-time are related to its $3 D$ gradient and time derivative by

$$
\mathbb{\nabla} \mathbf{f f}=(\nabla \mathrm{ff}, \partial \mathrm{ff} / \partial t) \quad \text { and } \quad \mathbb{D} \mathbb{I V f f}=\operatorname{div} \mathbf{f}+\partial f / \partial t .
$$

In particular, if $\mathrm{ff}$ is a vector field, the $4 \times 4$ matrix $\mathbb{\nabla} \mathrm{ff}$ admits the block decomposition ${ }^{3}$ :

$$
\mathbb{\nabla} \mathbf{f f}=\left(\begin{array}{cc}
\nabla \mathbf{f} & (\partial \mathbf{f} / \partial t)^{T} \\
\nabla f & \partial f / \partial t
\end{array}\right)
$$

Applying this block decomposition to the gradient of $\boldsymbol{X}$ gives

$$
\mathbb{F}:=\mathbb{\nabla} \mathcal{X}=\left(\begin{array}{cc}
\mathbf{F} & \mathbf{v} \\
\mathbf{0} & 1
\end{array}\right)
$$

\footnotetext{
${ }^{3}$ When defining matrices, we identify any vector with the corresponding row matrix.
} 
and we can remark that $\mathbb{J}:=\operatorname{det} \mathbb{F}$ coincides with $J:=\operatorname{det} \mathbf{F}$.

Applying the chain rule to the identity $\chi^{-1} \circ \chi=I d$ we get $\partial \chi^{-1} / \partial t=$ $-\mathbf{F}^{-1} \cdot \mathbf{v}$ and consequently

$$
\mathbb{F}^{-1}=\left(\begin{array}{cc}
\mathbf{F}^{-1} & \left(-\mathbf{F}^{-1} \cdot \mathbf{v}\right)^{T} \\
\mathbf{0} & 1
\end{array}\right), \quad \mathbb{F}^{-T}=\left(\begin{array}{cc}
\mathbf{F}^{-T} & \mathbf{0} \\
-\mathbf{F}^{-1} \cdot \mathbf{v} & 1
\end{array}\right) .
$$

Let $\mathrm{ff}=(\mathbf{f}, f)$ be of order $k \geq 1$. Equation (4) reads

$$
\begin{array}{r}
\operatorname{div}\left(J \mathbf{f}^{@} \cdot \mathbf{F}^{-T}-J f @ \otimes \mathbf{F}^{-1} \cdot \mathbf{v}\right)+\frac{\partial(J f @)}{\partial t} \\
=J\left(\operatorname{div} \mathbf{f}+\frac{\partial f}{\partial t}\right)^{@}
\end{array}
$$

which implies (24). Similarly, Eq. (5) becomes (25). It is then easy to prove that using (25) in (24) gives (26). We notice that equations (26) encompass the classical relationships between material and Eulerian derivatives.

The singularity surface $\mathbb{S}_{a}$ admits the parametrization $\Phi$ defined by $\Phi(\mathbf{s}, t)=(\varphi(\mathbf{s}, t), t)$ on the set $\Omega \times(0, T)$. A $4 \mathrm{D}$ vector $(\mathbf{m}, m)$ is orthogonal to $\mathbb{S}_{a}$ if it is orthogonal to the three tangent vectors $\partial_{1} \Phi=\left(\tau_{1}, 0\right)$, $\partial_{2} \Phi=\left(\tau_{2}, 0\right)$ and $\partial \Phi / \partial t=(\mathbf{w}, 1)$; that is if

$$
\tau_{1} \cdot \mathbf{m}=0, \quad \tau_{2} \cdot \mathbf{m}=0, \quad \mathbf{w} \cdot \mathbf{m}+m=0 .
$$

From the two first equalities we deduce that $\mathbf{m}$ is proportional to the normal $\mathbf{N}_{a}$ to $S_{a}$. Hence the vectors orthogonal to $\mathbb{S}_{a}$ are those proportional to $\mathbb{M}_{a}=\left(\mathbf{N}_{a},-c_{a}\right)$.

Noticing that the three vectors

$$
\mathbb{T}_{1}=\left(\tau_{1}, 0\right), \quad \mathbb{T}_{2}=\left(\tau_{2}, 0\right), \quad \mathbb{T}_{3}=\left(c_{a} \mathbf{N}_{a}, 1\right)
$$

span the tangent hyper-plane to $\mathbb{S}_{a}$ and applying point (i) of Proposition 3 to these vectors gives (27).

We notice that $\mathbb{M}_{b}:=\left(\mathbf{N}_{b},-c_{b}\right)$ is orthogonal to $\mathbb{S}_{b}$ so that point (iii) of Proposition 3

$$
\left[\left|\mathbb{J}^{-1} \mathbb{F}^{T}\right|\right] \cdot \mathbb{M}_{b}^{@}=\left[\left|\left(J^{-1} \mathbf{F}^{T} \cdot \mathbf{N}_{b}^{@}, J^{-1}\left(\mathbf{v} \cdot \mathbf{N}_{b}^{@}-c_{b}^{@}\right)\right)\right|\right]=0,
$$

which implies (28).

Point (iv) of Proposition 3 states that 
$\mathbb{J}^{-1} \mathbb{F}^{T} \cdot \mathbb{M}_{b}^{@}=\left(J^{-1} \mathbf{F}^{T} \cdot \mathbf{N}_{b}^{@}, J^{-1}\left(\mathbf{v} \cdot \mathbf{N}_{b}^{@}-c_{b}^{@}\right)\right)$ is orthogonal to $\mathbb{S}_{a}$ and so co-linear to $\left(\mathbf{N}_{a},-c_{a}\right)$. This implies

$$
\mathbf{N}_{a}=\frac{J^{-1} \mathbf{F}^{T} \cdot \mathbf{N}_{b}^{@}}{\left\|J^{-1} \mathbf{F}^{T} \cdot \mathbf{N}_{b}^{@}\right\|} \quad \text { and } \quad c_{a}=\frac{J^{-1}\left(c_{b}^{@}-\mathbf{v} \cdot \mathbf{N}_{b}^{@}\right)}{\left\|J^{-1} \mathbf{F}^{T} \cdot \mathbf{N}_{b}^{@}\right\|} .
$$

Equations (30) are the symmetrical relationships obtained by considering $\boldsymbol{X}^{-1}$.

Finally let us apply the rank-one property (11) for the jump of the gradient of the map $\mathbb{X}$. It states the existence of a $4 \mathrm{D}$ vector $\mathbb{U}=(\mathbf{u}, u)$, such that

$$
[|\mathbb{F}|]=\left(\begin{array}{cc}
{[|\mathbf{F}|]} & {\left[\left|\mathbf{v}^{T}\right|\right]} \\
\mathbf{0} & 0
\end{array}\right)=\mathbb{U} \otimes \mathbb{M}_{a}
$$

the space-time decomposition of which gives

$$
[|\mathbf{F}|]=\mathbf{u} \otimes \mathbf{N}_{a} \quad \text { and } \quad[|\mathbf{v}|]=-\mathbf{u} c_{a} .
$$

Eliminating $\mathbf{u}$ gives the following jump condition on $S_{a}(t)$ :

$$
c_{a}[|\mathbf{F}|]=-[|\mathbf{v}|] \otimes \mathbf{N}_{a} .
$$

Replacing in this equation $\mathbf{N}_{a}$ and $c_{a}$ by formulas (40) gives (29).

\section{Balance Equations and Corresponding Jump Conditions in the Space-Time}

Any balance equation for a quantity $f$ on $\mathbb{B}_{b}$ is of the type $\operatorname{div} \mathbf{f}+\partial f / \partial t=r$, where $\mathbf{f}$ is the corresponding flux and $r$ is a source term. This equation is written in the time-space (see Eq. (32)), introducing the 4D field defined on $\mathbb{B}_{b}$ by $\mathrm{ff}=(\mathbf{f}, f)$, in the simple form

$$
\mathbb{D I V} \mathbb{f f}=r,
$$

Owing to proposition 1 this balance equation is easily transported on $\mathbb{B}_{a}$ in the form $\mathbb{D} \mathbb{I V}\left(\mathbb{d} \mathbb{f f}^{@} \cdot \mathbb{F}^{-T}\right)=\mathbb{J} r{ }^{@}$ which reads

$$
\operatorname{div}\left(J \mathbf{f}^{@} \cdot \mathbf{F}^{-T}-J f^{@} \otimes \mathbf{F}^{-1} \cdot \mathbf{v}\right)+\frac{\partial\left(J f f^{@}\right)}{\partial t}=J r^{\circledR} .
$$

The jump condition on $\mathbb{S}_{b}$ associated to this balance equation is easily recovered by considering equation (42) in the sense of distributions. If we do 
not consider any surface source term, this jump condition reads $\left[\left|f \cdot \mathbb{M}_{b}\right|\right]=0$ which, recalling that $\mathbb{M}_{b}=\left(\mathbf{N}_{b},-c_{b}\right)$, reduces to the more usual equation

$$
\left[\left|\mathbf{f} \cdot \mathbf{N}_{b}-f c_{b}\right|\right]=0 .
$$

This jump condition is easily transported on $S_{a}$ : it takes the form $\left[\left|\mathbf{f}^{@} \cdot \mathbf{N}_{b}^{@}-f^{@} c_{b}^{@}\right|\right]=0$ which recalling (30) also reads

$$
\left[\left|J \mathbf{f}^{@} \cdot\left(\mathbf{F}^{-T} \cdot \mathbf{N}_{a}\right)-J f^{@} \otimes\left(\mathbf{F}^{-1} \cdot \mathbf{v}\right) \cdot \mathbf{N}_{a}-J c_{a} f @\right|\right]=0 .
$$

\section{Porous Medium with a Solid-Material Surface Singularity}

\subsection{Kinematics}

As we intend to give a macroscopic description of a porous medium, we consider a continuum made by the superposition of two continuous phases: a fluid one and a solid one.

Let us introduce the domains $B_{s} \subset \mathbb{R}^{3}$ and $B_{f} \subset \mathbb{R}^{3}$ (usually referred to as the Lagrangian configurations of the two constituents) and the maps

$$
\chi_{s}: \mathbb{B}_{s}:=B_{s} \times(0, T) \rightarrow \mathbb{R}^{3}, \quad \text { and } \quad \chi_{f}: \mathbb{B}_{f}:=B_{f} \times(0, T) \rightarrow \mathbb{R}^{3}
$$

which represent the placement of the solid and fluid constituents. The motion of the fluid inside the solid matrix is described by the function $\phi_{s}: \mathbb{B}_{s} \rightarrow B_{f}$ which, at any time $t$, associates to each solid particle $\mathbf{X}_{s}$ that particular fluid material particle $\mathbf{X}_{f}=\phi_{s}\left(\mathbf{X}_{s}, t\right)$ occupying the same physical position as $\mathbf{X}_{s}$. The three introduced maps are related by $\chi_{s}=\chi_{f} \circ \phi_{s}$. We can assume, extending $B_{s}$ and $\phi_{s}$ if necessary, that $\phi_{s}$ is an homeomorphism from $B_{s}$ to $B_{f}$. This extension and the resulting extension of $\chi_{s}$ have no physical sense, but make easier the description of open porous media. It will be mandatory to check that our final equations do not depend on the choice of this extension. Therefore $\chi_{s}\left(B_{s}, t\right)=\chi_{f}\left(B_{f}, t\right)$ and we denote $B_{e}(t)$ this time-varying $3 \mathrm{D}$ domain referred to as the Eulerian configuration. In the sequel, in order to apply our previous results, we assume that the $4 \mathrm{D}$-counterparts of $\chi_{s}, \chi_{f}$ and $\phi_{s}$ are piecewise $C^{1}$-diffeomorphisms.

We still adopt the superscript notation (s) (respectively (f) and (e) ) to denote the transport of a tensor field from the configuration where it is defined to $B_{s}$ (resp. $B_{f}, B_{e}$ ). For instance, if a tensor $\mathbf{t}$ is defined on $B_{f}$, then $\mathbf{t}^{\circledR}:=\mathbf{t} \circ \phi_{s}$, while if it is an Eulerian field defined on $B_{e}$, then $\mathbf{t}^{\circledR}:=\mathbf{t} \circ \chi_{s}$. 
We denote the space gradient of the three placements by

$$
\mathbf{F}_{s}:=\nabla \chi_{s}, \quad \mathbf{F}_{f}:=\nabla \chi_{f}, \quad \mathbf{G}_{s}:=\nabla \phi_{s},
$$

and its determinant by

$$
J_{s}:=\operatorname{det} \mathbf{F}_{s}, \quad J_{f}:=\operatorname{det} \mathbf{F}_{f}, \quad I_{s}:=\operatorname{det} \mathbf{G}_{s}
$$

It is immediate to check, that the chain rule gives $\mathbf{F}_{s}=\mathbf{F}_{f}^{(\subseteq} \cdot \mathbf{G}_{s}$ and $J_{s}=$ $J_{f}^{(s)} I_{s}$.

We define now the classical Lagrangian velocity fields $\mathbf{v}_{s}$ and $\mathbf{v}_{f}$, associated to the motion of the solid and of the fluid constituent, on $B_{s}$ and $B_{f}$ and, on $B_{s}$, the time derivative $\mathbf{u}_{s}$ of the map $\phi_{s}$, which is not a velocity in the classical sense, but plays a central role in further calculations:

$$
\mathbf{v}_{s}:=\frac{\partial \chi_{s}}{\partial t}, \quad \mathbf{v}_{f}:=\frac{\partial \chi_{f}}{\partial t}, \quad \mathbf{u}_{s}:=\frac{\partial \phi_{s}}{\partial t} .
$$

By the chain rule we get

$$
0=\frac{\partial}{\partial t}\left(\phi_{s}\left(\phi_{s}^{-1}\left(\mathbf{X}_{f}, t\right), t\right)\right)=\mathbf{G}_{s}^{\oplus} \cdot \frac{\partial \phi_{s}^{-1}}{\partial t}+\mathbf{u}_{s}^{\oplus} .
$$

This relationship allows us to link $\mathbf{v}_{s}, \mathbf{v}_{f}$ and $\mathbf{u}_{s}$ :

$$
\begin{aligned}
\mathbf{v}_{f} & =\frac{\partial \chi_{f}}{\partial t}=\frac{\partial}{\partial t}\left(\chi_{s}\left(\phi_{s}^{-1}\left(\mathbf{X}_{f}, t\right), t\right)\right)=\mathbf{F}_{s}^{\oplus} \cdot \frac{\partial \phi_{s}^{-1}}{\partial t}+\mathbf{v}_{s}^{\oplus} \\
& =-\mathbf{F}_{s}^{(\oplus)} \cdot\left(\mathbf{G}_{s}^{(\oplus)}\right)^{-1} \cdot \mathbf{u}_{s}^{\oplus}+\mathbf{v}_{s}^{\oplus} .
\end{aligned}
$$

Transporting this relationship on $B_{s}$ gives

$$
\left.\mathbf{v}_{f}^{(}\right)=\mathbf{v}_{s}-\mathbf{F}_{s} \cdot \mathbf{G}_{s}^{-1} \cdot \mathbf{u}_{s} .
$$

Let us define the acceleration fields $\gamma_{s}, \gamma_{f}$ and $\mathbf{a}_{s}$ as the time derivatives of $\mathbf{v}_{s}, \mathbf{v}_{f}$ and $\mathbf{u}_{s}$ respectively. Using Eq. (26) for the diffeomorphism $\phi_{s}$, it is straightforward that

$$
\frac{\partial}{\partial t} \mathbf{v}_{f}^{(ङ)}=\gamma_{f}^{(}+\nabla \mathbf{v}_{f}^{\Im} \cdot \mathbf{G}_{s}^{-1} \cdot \mathbf{u}_{s}
$$

Finally, since it is needed for further calculations, we compute the time derivative of the tensor $\mathbf{F}_{s} \cdot \mathbf{G}_{s}^{-1}$; using (26) for the map $\phi_{s}$ it is straightforward to recover that

$$
\begin{aligned}
\frac{\partial}{\partial t}\left(\mathbf{F}_{s} \cdot \mathbf{G}_{s}^{-1}\right) & =\frac{\partial}{\partial t} \mathbf{F}_{f}^{\Im}=\nabla \mathbf{F}_{f}^{\Im} \cdot \mathbf{G}_{s}^{-1} \cdot \mathbf{u}_{s}+\left(\nabla \mathbf{v}_{f}\right)^{\circledR} \\
& =\nabla\left(\mathbf{F}_{s} \cdot \mathbf{G}_{s}^{-1}\right) \cdot \mathbf{G}_{s}^{-1} \cdot \mathbf{u}_{s}+\nabla \mathbf{v}_{f}^{\Im} \cdot \mathbf{G}_{s}^{-1} .
\end{aligned}
$$


In the sequel we focus on a surface $S_{s}(t)$ which may be a surface of singularity for $\chi_{s}$ and/or $\phi_{s}$ and on the image surfaces $S_{f}(t)=\phi_{s}\left(S_{s}(t), t\right)$ and $S_{e}(t)=\chi_{s}\left(S_{s}(t), t\right)=\chi_{f}\left(S_{f}(t), t\right)$ which are moving surfaces in $B_{f}$ and $B_{e}(t)$. We apply to these surfaces the notations and formulas stated in the previous sections. In particular, we introduce the celerities $c_{s}, c_{f}$ and $c_{e}$ of $S_{s}(t), S_{f}(t)$ and $S_{e}(t)$ respectively.

Actually, in this paper we only consider the case in which the surface $S_{s}$ is a solid-material surface discontinuity which means that it does not depend on time. From now on we assume that $S_{s}$ is parametrized by a function $\varphi$ which does not depend on time. Consequently, the celerity $c_{s}$ of the surface $S_{s}$ is vanishing. This particular case of solid-material surface has many applications. It models all those phenomena in which $S_{s}$ divides the solid skeleton in two parts with different mechanical properties (e.g. different porosities, rigidities, etc.) It also models, as a limit case, the boundary of a fluid-filled porous matrix in contact with a pure fluid.

The hypothesis that the surface is solid-material $\left(c_{s}=0\right)$, applying (41) to both $\chi_{s}$ and $\phi_{s}$, implies

$$
\left[\left|\mathbf{v}_{s}\right|\right]=0 \quad \text { and } \quad\left[\left|\mathbf{u}_{s}\right|\right]=0 \quad \text { on } S_{s} .
$$

We underline that these equations do not imply $\left[\left|\mathbf{v}_{f}\right|\right]=0$.

We finally remark that if $\left(\mathbf{v}_{s}^{\ominus}-\mathbf{v}_{f}^{\ominus}\right) \cdot \mathbf{N}_{e}=0$, or equivalently by $(50)$ and (30) $\mathbf{u}_{s} \cdot \mathbf{N}_{f}^{\Im}=0$, then from (27)

$$
\left[\left|\mathbf{F}_{f}^{(} \cdot \mathbf{u}_{s}\right|\right]=\left[\left|\mathbf{v}_{s}-\mathbf{v}_{f}^{(}\right|\right]=0 .
$$

\subsection{Balance of Masses}

The masses $\mathcal{M}_{s}(B)$ and $\mathcal{M}_{f}(B)$ of solid skeleton and fluid contained in a part $B \subset B_{e}$ of the physical space at time $t$ are represented by means of two Eulerian densities $\rho_{s}$ and $\rho_{f}$ respectively in the form

$$
\mathcal{M}_{s}(B)=\int_{B} \rho_{s}, \quad \mathcal{M}_{f}(B)=\int_{B} \rho_{f} .
$$

These densities are usually called "apparent densities" and they do not coincide with the mass densities of the materials which constitute the solid skeleton or the fluid. Simple changes of variables give

$$
\begin{aligned}
& \mathcal{M}_{s}(B)=\int_{\chi_{s}^{-1}(B)} \rho_{s}^{\Im} J_{s} \text { and } \\
& \mathcal{M}_{f}(B)=\int_{\chi_{s}^{-1}(B)} \rho_{f}^{(} J_{s}=\int_{\chi_{f}^{-1}(B)} \rho_{f}^{\oplus} J_{f}, .
\end{aligned}
$$


which leads us to introduce the "solid-Lagrangian apparent densities" $\eta_{s}$, $m_{f}$ for the solid and the fluid constituent defined on $B_{s}$ by $\eta_{s}:=J_{s} \rho_{s}^{\S}$, $m_{f}:=J_{s} \rho_{f}^{\subseteq}$ and the "fluid-Lagrangian apparent density" $\eta_{f}$ of the fluid constituent on $B_{f}$ by $\eta_{f}:=J_{f} \rho_{f}^{\oplus}$. The densities $m_{f}$ and $\eta_{f}$ are related by $m_{f}=I_{s} \eta_{f}^{(s)}$

As we do not intend to model melting, dissolution or erosion phenomena, we assume conservation of mass for each constituent. Mass conservation for the solid skeleton and the fluid take the form of the balance laws

$$
\frac{\partial}{\partial t} \eta_{s}=0, \quad \text { and } \quad \frac{\partial}{\partial t} \eta_{f}=0
$$

which are of the type studied in section 5 . The results of section 5 give the associated jump conditions $\left[\left|\eta_{s}\right|\right] c_{s}=0$ and $\left[\left|\eta_{f}\right|\right] c_{f}=0$ on $S_{s}$ and $S_{f}$ respectively. As $c_{s}=0$ the first equation is trivially satisfied. So is is the second one if one assumes (which, as it is well known, can be done for a fluid without loss of generality) that $\eta_{f}$ is constant in space and time. However, the pull-back on $B_{s}$ of the fluid balance and jump equations, using the transport formulas (43) and (45) together with equation (50) gives the non-trivial equations

$$
\begin{aligned}
& \dot{m}_{f}+\operatorname{div} \mathbf{D}=\mathbf{0} \quad \text { on } B_{s}, \\
& {[|\mathbf{D}|] \cdot \mathbf{N}_{s}=0 \quad \text { on } S_{s},}
\end{aligned}
$$

where $\mathbf{D}:=-m_{f} \mathbf{G}_{s}^{-1} \cdot \mathbf{u}_{s}$ and $\dot{m}_{f}:=\partial m_{f} / \partial t$. The vector $\mathbf{D}$ is interpreted as the mass fluid flux through the porous medium in the Lagrangian configuration of the skeleton. The quantity $\mathbf{D} \cdot \mathbf{N}_{s}$, which is well defined at the interface $S_{s}$, is the flux (per unit area of $S_{s}$ ) of fluid flowing through the interface. We introduce

$$
\begin{aligned}
d & :=\left(\frac{\mathbf{D} \cdot \mathbf{N}_{s}}{\left\|J_{s} \mathbf{F}_{s} \cdot \mathbf{N}_{s}\right\|}\right)^{\ominus}=\rho_{f}\left(\mathbf{v}_{f}^{\ominus}-\mathbf{v}_{s}^{\ominus}\right) \cdot \mathbf{N}_{e} \\
& =\eta_{f}^{\ominus}\left\|\left(J_{f}^{-1} \mathbf{F}_{f}^{T}\right)^{\ominus} \cdot \mathbf{N}_{e}\right\|\left(\mathbf{N}_{f} \cdot \mathbf{u}_{s}\right)^{\ominus}
\end{aligned}
$$

which is well defined at the interface $S_{e}$ and corresponds to the flux (per unit area of $S_{e}$ ) of fluid flowing through the interface. 


\section{Evolution Equations and Associated Jump Conditions in Presence of Dissipation}

\subsection{Action and Rayleigh Functionals}

Recall that the kinematics of the considered porous medium is described by means of the fields $\chi_{s}$ and $\phi_{s}$ defined on $B_{s}$.

We introduce the kinetic energy

$$
\frac{1}{2} \int_{B_{e}}\left(\rho_{s}\left(\mathbf{v}_{s}^{\ominus}\right)^{2}+\rho_{f}\left(\mathbf{v}_{f}^{\ominus}\right)^{2}\right)=\int_{B_{s}} \Lambda\left(\eta_{s}, m_{f}, \mathbf{v}_{s}, \mathbf{v}_{f}^{(s)}\right)
$$

where $\Lambda\left(\eta_{s}, m_{f}, \mathbf{v}_{s}, \mathbf{v}_{f}^{(s)}\right)=1 / 2\left(\eta_{s}\left(\mathbf{v}_{s}\right)^{2}+m_{f}\left(\mathbf{v}_{f}^{(s}\right)^{2}\right)$ is the solid-Lagrangian pull-back of the kinetic energy density.

We now assume that the potential energy of the porous medium is characterized by a local density $\Psi$ on $B_{s}$ which depends on the kinematic descriptors $\chi_{s}$ and $\phi_{s}$ through the placement $\chi_{s}$, the strain tensor $\varepsilon:=$ $1 / 2\left(\mathbf{F}_{s}^{T} \cdot \mathbf{F}_{s}-\mathbf{I}\right)$ and the quantity of fluid contained in the porous medium $m_{f}=I_{s} \eta_{f}$. For instance $\Psi$ can be the sum of a non-homogeneous deformation energy potential $\Psi_{i}\left(\varepsilon, m_{f}, \mathbf{X}_{s}\right)$ and a potential accounting for external body forces $\Psi_{g}=\left(\eta_{s}+m_{f}\right) E_{p}\left(\chi_{s}\left(\mathbf{X}_{s}\right)\right)$. As we do not intend to model surface tension phenomena, we do not consider any concentration of energy on the singularity surface $S_{s}$. Neither do we consider any dependence of $\Psi$ on higher gradients of the kinematical fields as done for instance in Sciarra et al. (2008).

Setting $\mathbb{B}_{s}:=B_{s} \times(0, T)$, we define the action functional $\mathcal{A}$ for the porous system as

$$
\mathcal{A}:=\int_{\mathbb{B}_{s}}(\Lambda-\Psi) .
$$

It is well known that, in absence of dissipation, imposing the stationarity of the action implies that the kinematic descriptors satisfy the virtual power principle i.e. a weak form of the balance of momentum. As we want to account for dissipation phenomena, we introduce a generalized Rayleigh dissipation pseudo-potential $\mathcal{R}$ on the Eulerian configuration. In linear thermodynamics the dissipation $2 \mathcal{R}$ is a quadratic form of the velocity fields

$$
\begin{aligned}
2 \mathcal{R} & =\int_{B_{e} \backslash S_{e}} \mathcal{D}\left(\mathbf{v}_{s}^{\ominus}-\mathbf{v}_{f}^{\ominus}, \nabla\left(\mathbf{v}_{s}^{\ominus}\right), \nabla\left(\mathbf{v}_{f}^{\ominus}\right)\right) \\
& +\int_{S_{e}} \mathcal{D}_{S}\left(\left(\mathbf{v}_{s}^{\ominus}\right)^{-},\left(\mathbf{v}_{s}^{\ominus}\right)^{+},\left(\mathbf{v}_{f}^{\ominus}\right)^{-},\left(\mathbf{v}_{f}^{\ominus}\right)^{+}\right)
\end{aligned}
$$

where the volume density $\mathcal{D}$ is a positive quadratic form, the surface density $\mathcal{D}_{S}$ is a Galilean invariant quadratic form (the coefficients of these two forms 
may also depend on all the static parameters). In the sequel, we limit ourselves to the case

$$
\begin{gathered}
\mathcal{D}=\left(\mathbf{v}_{s}^{\ominus}-\mathbf{v}_{f}^{\ominus}\right) \cdot \mathbf{K} \cdot\left(\mathbf{v}_{s}^{\ominus}-\mathbf{v}_{f}^{\ominus}\right)+\nabla\left(\mathbf{v}_{s}^{\ominus}-\mathbf{v}_{f}^{\ominus}\right): \mathbf{B}: \nabla\left(\mathbf{v}_{s}^{\ominus}-\mathbf{v}_{f}^{\ominus}\right) \\
+\nabla \mathbf{v}_{f}^{\ominus}: \mathbf{M}: \nabla \mathbf{v}_{f}^{\ominus} \\
\mathcal{D}_{S}=\left[\left|\mathbf{v}_{f}^{\ominus}\right|\right] \cdot \mathbf{S} \cdot\left[\left|\mathbf{v}_{f}^{\ominus}\right|\right]
\end{gathered}
$$

where $\mathbf{K}$ and $\mathbf{S}$ are second order symmetric, positive tensors, $\mathbf{M}$ and $\mathbf{B}$ are symmetric positive fourth order tensors, the symbol : stands for the double contraction product. The tensor $\mathbf{K}$ accounts for the Darcy dissipation; its inverse (if invertible) is called the Darcy permeability tensor. The tensor $\mathbf{B}$ accounts for Brinkman dissipation. Classical fluid viscous effects are described by $\mathbf{M}$, while $\mathbf{S}$ describes friction effects at the interface. We already noticed that, as the extension of $\chi_{s}$ is arbitrary in a pure fluid region, the model should not depend on $\mathbf{v}_{s}$ in this region. Hence, $\mathbf{K}$ and $\mathbf{B}$ have to vanish in any pure fluid region.

We introduce respectively, the Darcy friction force $\kappa$, the Brinkman stress tensor $\Pi$, the fluid viscous stress tensor $\Pi_{f}$ and the friction surface force $\sigma$ as

$$
\begin{array}{ll}
\kappa:=\mathbf{K} \cdot\left(\mathbf{v}_{s}^{\ominus}-\mathbf{v}_{f}^{\ominus}\right), & \Pi:=\mathbf{B}: \nabla\left(\mathbf{v}_{s}^{\ominus}-\mathbf{v}_{f}^{\ominus}\right), \\
\Pi_{f}:=\mathbf{M}: \nabla \mathbf{v}_{f}^{\ominus}, & \sigma:=\mathbf{S} \cdot\left[\left|\mathbf{v}_{f}^{(}\right|\right] .
\end{array}
$$

This Rayleigh dissipation is pulled back on $B_{s}$ by simply changing the variables

$$
2 \mathcal{R}=\int_{B_{s} \backslash S_{s}} J_{s} \mathcal{D}^{(s)}+\int_{S_{s}} \mathcal{D}_{S}^{(s)}\left\|J_{s} \mathbf{F}_{s}^{-T} \cdot \mathbf{N}_{s}\right\| .
$$

In the application of this formula, the change of variables in $\mathcal{D}_{S}$ and in the first term of $\mathcal{D}$ is straightforward. The terms involving gradients in $\mathcal{D}$ need to be transported according to formula (2).

\subsection{Equations of Motion}

Let us denote by $\mathbf{q}:=\left(\chi_{s}, \phi_{s}\right)$ the kinematic descriptor of the medium (a field defined on $\mathbb{B}_{s}$ ). Hence the action $\mathcal{A}$ is a functional of $\mathbf{q}$. Moreover, let us denote by $\mathbf{q}_{t}, \dot{\mathbf{q}}_{t}$ the fields defined at any instant $t$ on $B_{s}$ by $\mathbf{q}_{t}\left(\mathbf{X}_{s}\right):=$ $\mathbf{q}\left(\mathbf{X}_{s}, t\right)$ and $\dot{\mathbf{q}}_{t}\left(\mathbf{X}_{s}\right):=\partial \mathbf{q} / \partial t\left(\mathbf{X}_{s}, t\right)$. The Rayleigh potential $\mathcal{R}$ is at each instant $t$ a functional of $\left(\mathbf{q}_{t}, \dot{\mathbf{q}}_{t}\right)$. 
The physical principle which determines the motion of a system can be alternatively stated in the framework of second Newton's law (balance of momentum), of D'Alembert principle (weak formulation of momentum balance) or of Rayleigh-Hamilton principle. We adopt this last approach which reads

$$
\frac{\partial \mathcal{A}}{\partial \mathbf{q}} \cdot \delta \mathbf{q}=\int_{0}^{T}\left(\frac{\partial \mathcal{R}}{\partial \dot{\mathbf{q}}_{t}} \mid \delta \mathbf{q}_{t}\right) d t
$$

Here, $\partial \mathcal{A} / \partial \mathbf{q}$ and $\partial \mathcal{R} / \partial \dot{\mathbf{q}}_{t}$ must be understood in the sense of Frechet differentials. Considering $\delta \mathbf{q}=\left(\delta \chi_{s}, \delta \phi_{s}\right)$ with compact support included in $B_{s}$, we get after a long but straightforward calculation (cfr. Appendix B):

$$
\begin{aligned}
\delta \mathcal{A} & :=\frac{\partial \mathcal{A}}{\partial \mathbf{q}} \cdot \delta \mathbf{q} \\
& =\int_{\mathbb{B}_{s} \backslash \mathbb{S}_{s}}\left[-\left(\eta_{s} \gamma_{s}+m_{f} \gamma_{f}^{(s)}+\operatorname{div}\left(\mathbf{F}_{s} \cdot \frac{\partial \Psi}{\partial \varepsilon}\right)-\frac{\partial \Psi}{\partial \chi_{s}}\right] \cdot \delta \chi_{s}+\right. \\
& \int_{\mathbb{B}_{s} \backslash \mathbb{S}_{s}}\left[\mathbf{G}_{s}^{-T} \cdot m_{f}\left(\mathbf{F}_{s}^{T} \cdot \gamma_{f}^{(s)}+\nabla\left(\frac{\partial \Psi}{\partial m_{f}}\right)\right)\right] \cdot \delta \phi_{s} \\
& +\int_{\mathbb{S}_{s}}\left(\left[\left|\mathbf{v}_{f}^{\Im} \otimes \mathbf{D}-\mathbf{F}_{s} \cdot \frac{\partial \Psi}{\partial \varepsilon}\right|\right] \cdot \mathbf{N}_{s}\right) \cdot \delta \chi_{s} \\
& +\int_{\mathbb{S}_{s}}\left(\left[\mid \mathbf{G}_{s}^{-T} \cdot\left(m_{f}\left(\frac{1}{2}\left(\mathbf{v}_{f}^{(s)}\right)^{2}-\frac{\partial \Psi}{\partial m_{f}}\right) \mathbf{I}\right.\right.\right. \\
& -\mathbf{F}_{s}^{T} \cdot \mathbf{v}_{f}^{\circledR} \otimes \mathbf{D} \cdot \mathbf{N}_{s} \cdot \delta \phi_{s} .
\end{aligned}
$$

On the other hand, computation of the right hand side of (65), reads (cfr. Appendix C for details)

$$
\begin{aligned}
& \int_{\mathbb{B}_{s} \backslash \mathbb{S}_{s}}-\operatorname{div}\left(J_{s} \Pi_{f}^{(\subseteq} \cdot \mathbf{F}_{s}^{-T}\right) \cdot \delta \chi_{s}+\int_{\mathbb{S}_{s}}\left[\left|J_{s} \Pi_{f}^{(} \cdot \mathbf{F}_{s}^{-T} \cdot \mathbf{N}_{s}\right|\right] \cdot \delta \chi_{s}+ \\
& \int_{\mathbb{B}_{s} \backslash \mathbb{S}_{s}} \mathbf{G}_{s}^{-T} \cdot \mathbf{F}_{s}^{T} \cdot\left(J_{s} \kappa^{(s)}-\operatorname{div}\left(J_{s}\left(\Pi^{(s)}-\Pi_{f}^{(}\right)^{T} \cdot \mathbf{F}_{s}^{-T}\right)\right) \cdot \delta \phi_{s} \\
& +\int_{\mathbb{S}_{s}}\left[\mid J_{s} \mathbf{G}_{s}^{-T} \cdot \mathbf{F}_{s}^{T} \cdot\left(\left(\left(\Pi^{(}-\Pi_{f}^{(}\right)^{T}\right) \cdot \mathbf{F}_{s}^{-T} \cdot \mathbf{N}_{s}\right.\right. \\
& -\left\|J_{s} \mathbf{F}_{s}^{-T} \cdot \mathbf{N}_{s}\right\| \sigma^{(s)} \cdot \delta \phi_{s} \text {. }
\end{aligned}
$$

Considering arbitrary test functions $\delta \chi_{s}$ and $\delta \phi_{f}$ with compact support included in $\mathbb{B}_{s} \backslash \mathbb{S}_{s}$ the variational principle (65) implies the following system 
of equations valid in $\mathbb{B}_{s} \backslash \mathbb{S}_{s}$

$$
\begin{aligned}
& -\left(\eta_{s} \gamma_{s}+m_{f} \gamma_{f}^{\Im}\right)+\operatorname{div}\left(\mathbf{F}_{s} \cdot \frac{\partial \Psi}{\partial \varepsilon}\right)-\frac{\partial \Psi}{\partial \chi_{s}} \\
& =-\operatorname{div}\left(J_{s} \Pi_{f}^{\Im} \cdot \mathbf{F}_{s}^{-T}\right), \\
& m_{f}\left(\mathbf{F}_{s}^{T} \cdot \gamma_{f}^{\Im}+\nabla\left(\frac{\partial \Psi}{\partial m_{f}}\right)\right) \\
& =\mathbf{F}_{s}^{T} \cdot\left(J_{s} \kappa^{\Im}-\operatorname{div}\left(J_{s}\left(\Pi^{\Im}-\Pi_{f}^{\Im}\right)^{T} \cdot \mathbf{F}_{s}^{-T}\right)\right) .
\end{aligned}
$$

Then, considering test functions with compact support which intersects $\mathbb{S}_{s}$, we get the jump conditions valid on $\mathbb{S}_{s}$ :

$$
\begin{aligned}
& {\left[\left|\mathbf{v}_{f}^{\Im} \otimes \mathbf{D}-\mathbf{F}_{s} \cdot \frac{\partial \Psi}{\partial \varepsilon}\right|\right] \cdot \mathbf{N}_{s}=\left[\left|J_{s} \Pi_{f}^{\circledast} \cdot \mathbf{F}_{s}^{-T}\right|\right] \cdot \mathbf{N}_{s},} \\
& {\left[\left|\mathbf{G}_{s}^{-T} \cdot\left(m_{f}\left(\frac{1}{2}\left(\mathbf{v}_{f}^{\Im}\right)^{2}-\frac{\partial \Psi}{\partial m_{f}}\right) \mathbf{I}-\mathbf{F}_{s}^{T} \cdot \mathbf{v}_{f}^{\Im} \otimes \mathbf{D}\right)\right|\right] \cdot \mathbf{N}_{s}} \\
& =\left[\mid \mathbf{G}_{s}^{-T} \cdot \mathbf{F}_{s}^{T} \cdot\left(J_{s}\left(\left(\Pi^{\Im}-\Pi_{f}^{\Im}\right)^{T}\right) \cdot \mathbf{F}_{s}^{-T} \cdot \mathbf{N}_{s}\right.\right. \\
& -\left\|J_{s} \mathbf{F}_{s}^{-T} \cdot \mathbf{N}_{s}\right\| \sigma^{(s)} \text {. }
\end{aligned}
$$

We remark that equations (68) and (69) encompass the well known Lagrangian balance equation for the total stress and the corresponding jump condition; these equations only involve physical quantities. As for equations (70), they are not available in the literature. It is not obvious that in their present form they are Galilean invariant, that they do not depend on the choice of the reference configuration of the fluid and that, when considering pure fluid regions, they do not depend on the extension of $\chi_{s}$ in this region.

\subsection{Galilean Invariance}

In this section we check that the set of evolution equations (68), together with the associated jump conditions (69), (70), respect Galilean invariance. In order to do so we just rewrite them in a new Galilean reference frame which moves with a constant velocity $\mathbf{v}_{0}$ with respect to the original one and we check that the system of equations do not change. We start by noticing that the dissipative terms appearing on the right hand sides of (68)-(70) are Galilean invariant since they only involve relative velocities or gradients of velocities.

As for the equations of motion (68) they are Galilean invariant since the accelerations $\gamma_{s}$ and $\gamma_{f}^{(\S)}$ do not change when changing the Galilean 
reference frame, as the relative velocity $\mathbf{v}_{0}$ is constant with respect to time. The invariance of the jump condition (69) is immediately verified simply noticing that $\left[\left|\mathbf{v}_{f}^{(s}\right|\right]=\left[\left|\mathbf{v}_{f}^{(s}-\mathbf{v}_{0}\right|\right]$ and that $\mathbf{D}$, being a relative velocity, is also Galilean invariant.

Equation (70) does not have an evident Galilean invariant form. However, let us prove that it is equivalent to

$$
\begin{aligned}
& {\left[\mid \mathbf{G}_{s}^{-T} \cdot\left(m_{f}\left(\frac{1}{2}\left(\mathbf{v}_{f}^{(}-\mathbf{v}_{s}\right)^{2}-\frac{\partial \Psi}{\partial m_{f}}\right) \mathbf{I}\right.\right.} \\
& -\mathbf{F}_{s}^{T} \cdot\left(\mathbf{v}_{f}^{(s}-\mathbf{v}_{s}\right) \otimes \mathbf{D} \cdot \mathbf{N}_{s} \\
& \left.=\left[\mid \mathbf{G}_{s}^{-T} \cdot \mathbf{F}_{s}^{T} \cdot\left(J_{s}\left(\left(\Pi^{(}\right)-\Pi_{f}^{(s)}\right)^{T}\right) \cdot \mathbf{F}_{s}^{-T} \cdot \mathbf{N}_{s}-\left\|J_{s} \mathbf{F}_{s}^{-T} \cdot \mathbf{N}_{s}\right\| \sigma^{(s)}\right)\right],
\end{aligned}
$$

in which only Galilean invariant quantities appear.

Indeed, the difference $Q$ between the left hand sides of (71) and (70), using continuity of $\mathbf{v}_{s}$ and recalling Eq. (50), reads

$$
\begin{aligned}
Q & =\frac{1}{2} \mathbf{v}_{s}^{2}\left[\left|m_{f} \mathbf{G}_{s}^{-T}\right|\right] \cdot \mathbf{N}_{s} \\
& +\left[\left|-m_{f}\left(\mathbf{v}_{f}^{(s)} \cdot \mathbf{v}_{s}\right) \mathbf{G}_{s}^{-T}+\mathbf{G}_{s}^{-T} \cdot \mathbf{F}_{s}^{T} \cdot \mathbf{v}_{s} \otimes \mathbf{D}\right|\right] \cdot \mathbf{N}_{s} .
\end{aligned}
$$

We know that, applying formula (28) to the map $\phi_{s}^{-1}$ under the assumption $\left[\left|\eta_{f}\right|\right]=0$, one gets

$$
\left[\left|m_{f} \mathbf{G}_{s}^{-T}\right|\right] \cdot \mathbf{N}_{s}=0 \text {. }
$$

Hence, using again the continuity of $\mathbf{v}_{s}$ it is easy to get

$$
Q=\mathbf{v}_{s} \cdot\left[\left|-m_{f} \mathbf{v}_{f}^{(s)} \otimes \mathbf{G}_{s}^{-T}+\mathbf{F}_{s} \cdot \mathbf{G}_{s}^{-1} \otimes \mathbf{D}\right|\right] \cdot \mathbf{N}_{s}
$$

which using the balance of mass (56) and again (72) is equivalent to

$$
Q=\mathbf{v}_{s} \cdot\left\{-\left[\left|\mathbf{v}_{f}^{(}\right|\right] \otimes m_{f} \mathbf{G}_{s}^{-T}+\left[\left|\mathbf{F}_{s} \cdot \mathbf{G}_{s}^{-1}\right|\right] \otimes \mathbf{D}\right\} \cdot \mathbf{N}_{s} .
$$

Moreover, by Eq. (30) applied to the map $\chi_{s}$ it is clear that $\mathbf{N}_{s}=\left(J_{s}^{-1} \mathbf{F}_{s}^{T} \cdot \mathbf{N}_{e}^{(}\right) /\left\|J_{s}^{-1} \mathbf{F}_{s}^{T} \cdot \mathbf{N}_{e}^{(}\right\|$, where $\mathbf{N}_{e}$ is the unit normal vector to the Eulerian surface $S_{e}$. Using this result and recalling the definitions of $m_{f}$ and $\mathbf{D}$, it can be recognized that

$$
\begin{aligned}
& Q=-\frac{\eta_{f}^{(s)} \mathbf{v}_{s}}{\left\|J_{s}^{-1} \mathbf{F}_{s}^{T} \cdot \mathbf{N}_{e}^{(s}\right\|} \cdot\left[\left|\mathbf{v}_{f}^{(s}\right|\right] I_{s} J_{s}^{-1}\left(\mathbf{F}_{s} \cdot \mathbf{G}_{s}^{-1}\right)^{T} \\
& +\frac{\eta_{f}^{\Im} \mathbf{v}_{s}}{\left\|J_{s}^{-1} \mathbf{F}_{s}^{T} \cdot \mathbf{N}_{e}^{(\subseteq}\right\|} \cdot I_{s} J_{s}^{-1}\left[\left|\mathbf{F}_{s} \cdot \mathbf{G}_{s}^{-1}\right|\right] \otimes\left(\mathbf{v}_{f}^{(}-\mathbf{v}_{s}\right) \cdot \mathbf{N}_{e}^{\Im} .
\end{aligned}
$$


Therefore, the quantity $Q$ vanishes owing to the purely kinematical formula (29) applied to the diffeomorphism $\chi_{s} \circ \phi_{s}^{-1}$ (notice that $\mathbf{v}_{s} \cdot \mathbf{N}_{e}^{\Im}=c_{e}^{\Im}$ ).

It follows that the Galilean invariance of the last jump condition remains proven.

\subsection{The equations do not depend on the choice of the fluid ref- erence configuration}

Equation (71) seems to depend on the choice of the fluid reference configuration through the tensor $\mathbf{G}_{s}$ (all others equations clearly do not depend on this choice). Let us first project this equation on the tangent plane to $S_{f}$. Hence, if $\tau_{e}$ is an arbitrary tangent vector to $S_{e}$, multiplication of equation (71) by $\mathbf{G}_{s} \cdot \mathbf{F}_{s}^{-1} \cdot \tau_{e}^{\Im}$ gives

$$
\begin{aligned}
& \tau_{e}^{(\Im)}\left[\left|\left(\mathbf{v}_{f}^{(}-\mathbf{v}_{s}\right)\right|\right] d^{(}+\tau_{e}^{\Im} \cdot\left[\left|\left(\Pi^{(}-\Pi_{f}^{(}\right)^{T}\right|\right] \cdot \mathbf{N}_{e}^{(} \\
& -\left\|J_{s} \mathbf{F}_{s}^{-T} \cdot \mathbf{N}_{s}\right\| \tau_{e}^{\Im} \cdot \mathbf{S} \cdot\left[\left|\mathbf{v}_{f}^{(}-\mathbf{v}_{s}\right|\right]=0
\end{aligned}
$$

Now let us project equation (71) in a different direction, multiplying it by the continuous quantity $-\mathbf{u}_{s}=\mathbf{G}_{s} \cdot \mathbf{F}_{s}^{-1} \cdot\left(\mathbf{v}_{f}^{()}-\mathbf{v}_{s}\right)$ : notice that, owing to (57) and (54), this projection gives additional information to (73) only if $d \neq 0$. We get

$$
\begin{aligned}
& {\left[\left|\left(-\frac{1}{2}\left(\mathbf{v}_{f}^{\Im}-\mathbf{v}_{s}\right)^{2}-\frac{\partial \Psi}{\partial m_{f}}\right)\right|\right] d^{\Im}} \\
& =\left[\left|\left(\mathbf{v}_{f}^{\Im}-\mathbf{v}_{s}\right) \cdot\left(\left(\left(\Pi^{\Im}-\Pi_{f}^{\Im}\right)^{T}\right) \cdot \mathbf{N}_{e}^{(}-\sigma^{\Im}\right)\right|\right] .
\end{aligned}
$$

Notice that this equation does not depend on the choice of the reference configuration of the fluid.

\section{The Case of a Deformable Porous Medium Surrounded by a Pure Fluid}

We now assume that the surface discontinuity $S_{s}$ separates a given porous medium (which occupies the volume $B_{s}^{+}$) from a pure fluid (which occupies the volume $B_{s}^{-}$). Proper evolution equations and boundary conditions are deduced from our previous results.

Clearly, the equations of motion of the porous medium in $B_{s}^{+}$coincide with (68) where the potential $\Psi$ is replaced by its restriction $\Psi^{+}$in $B_{s}^{+}$.

When the fluid is pure, its Eulerian energy density, its chemical potential and its pressure are functions of its mass density only. These three real 
functions are denoted respectively by $\Psi_{f}, \mu_{f}$ and $p_{f}$. They are related by

$$
\mu_{f}(y)=\Psi_{f}^{\prime}(y) \quad \text { and } \quad p_{f}(y)=-\Psi_{f}(y)+y \Psi_{f}^{\prime}(y),
$$

Therefore, the restriction $\Psi^{-}$of the potential $\Psi$ in $B_{s}^{-}$is that of a pure fluid: transporting the Eulerian density $\Psi_{f}\left(\rho_{f}\right)$ on $B_{s}$, we get

$$
\Psi^{-}\left(\varepsilon, m_{f}\right)=J_{s} \Psi_{f}\left(\rho_{f}^{\circledR}\right)=J_{s} \Psi_{f}\left(J_{s}^{-1} m_{f}\right)
$$

note that $J_{s}$ is a function of $\varepsilon$ only, as we have $J_{s}=\operatorname{det} \mathbf{F}_{s}=\operatorname{det} \sqrt{2 \varepsilon+\mathbf{I}}=$ $\sqrt{\operatorname{det}(2 \varepsilon+\mathbf{I})}$.

In the pure fluid region $B_{s}^{-}$we clearly have $\eta_{s}=0$ and, as already noticed, $\mathbf{K}^{\circledR}=0, \mathbf{M}^{\circledR}=0$. We also neglect external body forces and viscous forces by setting $\partial \Psi^{-} / \partial \chi_{s}=0$ and $\Pi=\Pi_{f}=0$.

With these assumptions, the equation of motion (68) in $B_{s}^{-}$reads

$$
\begin{aligned}
\left.m_{f} \gamma_{f}^{(}\right) & =\operatorname{div}\left(\mathbf{F}_{s} \cdot \frac{\partial \Psi^{-}}{\partial \varepsilon}\right) \\
& =\operatorname{div}\left[\mathbf{F}_{s} \cdot\left(\left.\Psi_{f}\right|_{\rho_{f}^{\circledast}} \frac{\partial J_{s}}{\partial \varepsilon}+\left.J_{s} \frac{\partial}{\partial \varepsilon} \Psi_{f}\right|_{\rho_{f}^{\circledast}}\right)\right] \\
& =\operatorname{div}\left[\mathbf{F}_{s} \cdot\left(\left.\Psi_{f}\right|_{\rho_{f}^{\circledast}} \frac{\partial J_{s}}{\partial \varepsilon}+\left.J_{s} \frac{\partial \Psi_{f}}{\partial \rho_{f}}\right|_{\rho_{f}^{\odot}} \frac{\partial}{\partial \varepsilon}\left(J_{s}^{-1} m_{f}\right)\right)\right] .
\end{aligned}
$$

Recalling that $\partial J_{s} / \partial \varepsilon=J_{s} \mathbf{F}_{s}^{-1} \cdot \mathbf{F}_{s}^{-T}$ and $\partial J_{s}^{-1} / \partial \varepsilon=-J_{s}^{-2} \partial J_{s} / \partial \varepsilon$, equation (77) can be simplified in

$$
m_{f} \gamma_{f}^{(}=\operatorname{div}\left[\left(\left.J_{s} \Psi_{f}\right|_{\rho_{f}^{\circledast}}-\left.m_{f} \mu_{f}\right|_{\rho_{f}^{\odot}}\right) \mathbf{F}_{s}^{-T}\right] ;
$$

or equivalently, recalling the inverse of Eq. (28) and (75), in

$$
m_{f} \gamma_{f}^{(}=-J_{s} \mathbf{F}_{s}^{-T} \cdot \nabla\left(\left.p_{f}\right|_{\rho_{f}^{\circledast}}\right) .
$$

This last equation, when transported on the Eulerian configuration, reads

$$
\nabla p_{f}+\rho_{f} \gamma_{f}^{\ominus}=0
$$

we thus recover the usual equation for the motion of a non-viscous fluid fluid.

Let us now study the second of equations (68) also valid in $B_{s}^{-}$. Indeed, the existence of an supplementary equation may seem astonishing. Under 
the hypotheses we formulated, recalling (76) and (75), Eq. (68) can be rewritten

$$
m_{f} \nabla\left(\left.\mu_{f}\right|_{\rho_{f}^{(\odot) ~}}\right)+m_{f} \mathbf{F}_{s}^{T} \cdot \gamma_{f}^{(s)}=0,
$$

which, multiplied on the left by $\mathbf{F}_{s}^{-T}$, gives

$$
J_{s} \rho_{f}^{(\subseteq} \mathbf{F}_{s}^{-T} \cdot \nabla\left(\left.\mu_{f}\right|_{\rho_{f}^{(ङ)}}\right)+m_{f} \gamma_{f}^{(S)}=0
$$

This last equation is clearly equivalent to Eq. (78) as $\left.\nabla\left(p_{f}\right)\right|_{\rho_{f}^{\Im}}=\left.\rho_{f}^{\Im} \nabla\left(\mu_{f}\right)\right|_{\rho_{f}^{\circledR}}$. Hence, as expected, the fluid is governed only by the usual equation for an non-viscous fluid.

Let us consider the jump condition given by Eq. (69) on the surface $S_{s}$ which divides the porous medium region $B_{s}^{+}$from the pure fluid region $B_{s}^{-}$. Considering the expression (76) for the potential of the pure fluid, it becomes

$$
\begin{aligned}
& \left(-\mathbf{F}_{s}^{+} \cdot\left(\frac{\partial \Psi}{\partial \varepsilon}\right)^{+}+\left(\mathbf{v}_{f}^{(s)}\right)^{+} \otimes \mathbf{D}\right) \cdot \mathbf{N}_{s} \\
& =\left(\left.J_{s}^{+}\left(\mathbf{F}_{s}^{+}\right)^{-T} p_{f}\right|_{\rho_{f}^{(ङ)}}+\left(\mathbf{v}_{f}^{()}\right)^{-} \otimes \mathbf{D}\right) \cdot \mathbf{N}_{s},
\end{aligned}
$$

where we used the fact that $J_{s}^{-} \mathbf{F}_{s}^{-} \cdot \mathbf{N}_{s}=J_{s}^{+} \mathbf{F}_{s}^{+} \cdot \mathbf{N}_{s}$ (here again the superscripts + and - indicate the traces on $S_{s}$ of quantities defined on $B_{s}^{+}$ and $B_{s}^{-}$respectively).

As for the second jump condition (70), it becomes

$$
\begin{aligned}
& \left(\mathbf{G}_{s}^{+}\right)^{-T} \cdot\left[\left(-m_{f}^{+} \frac{\partial \Psi^{+}}{\partial m_{f}^{+}}+\frac{1}{2} m_{f}^{+}\left(\mathbf{v}_{f}^{(s)}\right)^{+}{ }^{2}\right) \mathbf{I}\right. \\
& -\left(\mathbf{F}_{s}^{+}\right)^{T} \cdot\left(\mathbf{v}_{f}^{(s)}\right)^{+} \otimes \mathbf{D} \cdot \mathbf{N}_{s}= \\
& \left(\mathbf{G}_{s}^{-}\right)^{-T} \cdot\left[\left(\left(-\left.m_{f}^{-} \mu_{f}\right|_{\rho_{f}^{(}}+\frac{1}{2} m_{f}^{-}\left(\mathbf{v}_{f}^{(s)}\right)^{-} 2\right) \mathbf{I}\right.\right. \\
& -\left(\mathbf{F}_{s}^{-}\right)^{T} \cdot\left(\mathbf{v}_{f}^{(s)}\right)^{-} \otimes \mathbf{D} \cdot \mathbf{N}_{s} .
\end{aligned}
$$

In conclusion, the motion of the porous medium is driven by two independent equations of the type (68), while the motion of the pure fluid is driven by a simple equation in the form (78). The differential system is completed by two independent jump conditions given by (80) and (81). Equation (80) 
states that at a permeable interface between a porous medium and a pure fluid the jump of stress equals the negative of the jump of transported fluid momentum. Equation (81) states how at the same interface the jump of chemical potential plus fluid kinetic energy is related to transported fluid momentum.

\section{Conclusions}

To our knowledge, the inertia terms appearing in the jump condition $(69)_{2}$ are not found in the literature. Moreover all boundary conditions (69) are valid also when the solid matrix is suffering large deformations and when the Stokes fluid-flow condition is not applicable.

A deduction of the jump condition valid in the particular case of absence of inertia, of Darcy-Brinkman and Beavers-Joseph dissipation is presented in Baek and Srinivasa (2004).

Other authors (see e.g. Neale and Nader (1974), Vafai and Thiyagaraja (1987), Vafai and Kim (1990), Poulikakos and Kazmierczak (1997)) based themselves on the pioneering works of Beavers and Joseph (1967) and Saffman (1971) to justify the so-called slip boundary conditions at the interface between a porous matrix and an external viscous fluid. Beavers-JosephSaffman conditions include the continuity of the normal components of the relative velocity and of the gradient of the relative velocity at the interface between the porous medium and the external fluid: nevertheless, they only describe phenomena related to the viscosity of the outflowing fluid with no consideration of inertial effects and Darcy-Brinkman dissipation. BeaversJoseph-Saffman conditions can be deduced from jump condition (69), once assuming that the solid matrix is suffering small deformation and when Stokes fluid-flow condition is verified.

The jump conditions deduced in Deresiewicz (1960-1964), are suitable to assure that the differential problem of Darcy-Fillunger-Terzaghi-Biot is wellposed (see Fillunger (1936), Terzaghi (1943), Biot (1941-1963)): these last jump conditions can be obtained as a particular case from Beavers-JosephSaffman conditions, once it is possible to neglect dissipative phenomena at the considered interface. In Albers (2006), Wilmanski (1999-2006), De La Cruz et al. (1992), Quiroga-Goode and Carcione (1997) the jump conditions proposed by ? are used to study wave propagation phenomena at discontinuity surfaces in porous media.

In (Coussy and Bourbie (1984), Coussy et al. (1998), Rasolofosaon and Coussy (1985-1986), a variational approach is used to study some wave propagation phenomena of interest in oil industry: the boundary conditions proposed by Deresiewicz (1963) are examined there with a view towards the 
applications.

In Kubik and Cieszko (2005) a dissipative Rayleigh surface potential is proposed which is suitable to produce a particular form of Beavers-JosephSaffman boundary conditions: many versions of them are compared in Alazmi and Vafai (2001).

The jump conditions (69) also allow for describing phenomena in which inertial effects are relevant. The inertial terms here newly introduced are quadratic in the velocity fields at the interface: when Stokes fluid-flow conditions hold (and when the solid matrix is subject to "small deformations") they may be negligible. Indeed, when the equations are linearized in the neighborhood of a state of rest (i.e. when all velocity fields and their gradients are vanishing) the aforementioned inertial terms do not produce, in the resulting boundary conditions, any term additional to those appearing in Beavers-Joseph-Saffman conditions. However, when the linearization procedure is performed in the neighborhood of a state in which some velocity fields are not vanishing then inertial terms cannot be neglected, even when Rayleigh modeling hypothesis applies. Finally one should remark that in Ochoa-Tapia and Whitaker (1998) some inertial effects at the interface are considered. However, they deduce no-slip conditions for tangential velocity and a normal-to-the-interface boundary condition by means of an averaging procedure involving "excess quantities". Their assumptions produce inertial terms in which only the tangential part of the fluid velocity appears.

Future investigations will be aimed to get a generalization of the newly found boundary conditions to the case of shock waves.

\section{A Appendix: Some Preliminary Variations}

We now compute how the different quantities appearing in $\mathcal{A}$ vary when the two independent kinematical variables $\chi_{s}$ and $\phi_{s}$ change. We denote $\delta \chi_{s}$ and $\delta \phi_{s}$ the infinitesimal changes of $\chi_{s}$ and $\phi_{s}$ respectively.

Recall that we have assumed that the singularity surface $S_{s}$ (if any) of the fields $\chi_{s}$ and $\phi_{s}$ is fixed in $B_{s}$. Owing to this assumption, the fact of considering the fields $\delta \chi_{s}$ and $\delta \phi_{s}$ regular in $B_{s}$ is sufficient to explore the whole space of considered functions $\chi_{s}$ and $\phi_{s}$ i.e. the set of the $C_{p w}^{1}$ functions the singularity surface of which, if any, must be the surface $S_{s}$. This would not be the case if one wants to consider a moving singularity surface. Such a situation requires a more delicate treatment and will be developed in a forthcoming paper.

As far as the fields $\varepsilon, \eta_{s}$ and $\mathbf{v}_{s}$ attached to the solid constituent are concerned, their variation in terms of the independent kinematical fields 
are given by: ${ }^{4}$

$$
\begin{aligned}
& \delta \varepsilon=\frac{1}{2} \delta\left(\mathbf{F}_{s}^{T} \cdot \mathbf{F}_{s}-\mathbf{I}\right) \\
& =\frac{1}{2}\left(\delta \mathbf{F}_{s}^{T} \cdot \mathbf{F}_{s}+\mathbf{F}_{s}^{T} \cdot \delta \mathbf{F}_{s}\right)=\left(\mathbf{F}_{s}^{T} \cdot \nabla\left(\delta \chi_{s}\right)\right)^{s y m}, \\
& \delta \eta_{s}=0, \quad \delta \mathbf{v}_{s}=\delta \dot{\chi}_{s}
\end{aligned}
$$

We now compute the variation of the fields $m_{f}$ and $\mathbf{v}_{f}^{(s)}$ attached to the fluid constituent. In particular, if we want to compute $\delta m_{f}$ the preliminary variation of the fields $I_{s}$ and $\left.\eta_{f}^{(}\right)$must be established $^{5}$ :

$$
\begin{gathered}
\delta I_{s}=\delta\left(\operatorname{det}\left(\nabla \phi_{s}\right)\right)=I_{s} \mathbf{G}_{s}^{-T} \mid \nabla\left(\delta \phi_{s}\right) ; \\
\delta \eta_{f}^{()}=\delta\left(\eta_{f} \circ \phi_{s}\right)=\left(\nabla \eta_{f}\right)^{\Im} \cdot \delta \phi_{s}=\nabla \eta_{f}^{()} \cdot \mathbf{G}_{s}^{-1} \cdot \delta \phi_{s} .
\end{gathered}
$$

The variation $\delta m_{f}$ can be now computed as follows:

$$
\begin{aligned}
& \left.\delta m_{f}=\delta\left(I_{s} \eta_{f}^{(\S)}\right)=\eta_{f}^{(} \delta I_{s}+I_{s} \delta \eta_{f}^{(}\right) \\
& =I_{s}\left(\eta_{f}^{(s)} \mathbf{G}_{s}^{-T} \mid \nabla\left(\delta \phi_{s}\right)+\nabla \eta_{f}^{\Im} \cdot \mathbf{G}_{s}^{-1} \cdot \delta \phi_{s}\right) \\
& =\operatorname{div}\left(\eta_{f}^{(\Im)} I_{s} \mathbf{G}_{s}^{-1} \cdot \delta \phi_{s}\right)=\operatorname{div}\left(m_{f} \mathbf{G}_{s}^{-1} \cdot \delta \phi_{s}\right),
\end{aligned}
$$

where we used the fact that $\operatorname{div}\left(I_{s} \mathbf{G}_{s}^{-T}\right)=0$ proved in Eq. (5).

The variation of the solid Lagrangian fluid velocity $\mathbf{v}_{f}^{(s)}$ is now computed. Recalling Eq. (50) it is easy to show that ${ }^{6}$

$$
\begin{aligned}
\delta \mathbf{v}_{f}^{(s)} & =\delta \mathbf{v}_{s}-\delta \mathbf{F}_{s} \cdot \mathbf{G}_{s}^{-1} \cdot \mathbf{u}_{s}+\mathbf{F}_{s} \cdot \mathbf{G}_{s}^{-1} \cdot \delta \mathbf{G}_{s} \cdot \mathbf{G}_{s}^{-1} \cdot \mathbf{u}_{s}-\mathbf{F}_{s} \cdot \mathbf{G}_{s}^{-1} \cdot \delta \mathbf{u}_{s} \\
& =\delta \dot{\chi}_{s}-\nabla\left(\delta \chi_{s}\right) \cdot \mathbf{G}_{s}^{-1} \cdot \mathbf{u}_{s}+\mathbf{F}_{s} \cdot \mathbf{G}_{s}^{-1} \cdot \nabla\left(\delta \phi_{s}\right) \cdot \mathbf{G}_{s}^{-1} \cdot \mathbf{u}_{s} \\
& -\mathbf{F}_{s} \cdot \mathbf{G}_{s}^{-1} \cdot \delta \dot{\phi}_{s} .
\end{aligned}
$$

\footnotetext{
${ }^{4}$ Given a tensor field $\sigma, \sigma^{\text {sym }}:=\frac{1}{2}\left(\sigma+\sigma^{T}\right)$ denotes the symmetric part of $\sigma$. Moreover, we denote in the sequel the partial time derivatives of solid-Lagrangian fields by a superposed dot.

${ }^{5}$ We recall that, given a tensor field $\mathbf{M}$, the derivation rule for the determinant reads: $\delta(\operatorname{det} \mathbf{M})=\operatorname{det} \mathbf{M}\left(\mathbf{M}^{-T} \mid \delta \mathbf{M}\right)$.

${ }^{6}$ Given a matrix $\mathbf{G}$, the differentiation formula for its inverse $\mathbf{G}^{-1}$ reads $\delta\left(\mathbf{G}^{-1}\right)=$ $-\mathbf{G}^{-1} . \delta \mathbf{G} \cdot \mathbf{G}^{-1}$. This result is easily recovered differentiating the equality $\mathbf{G} \cdot \mathbf{G}^{-1}=\mathbf{I}$.
} 


\section{B Appendix: Variation of the Action Functional}

In order to suitably perform the solid-Lagrangian variation $\delta \mathcal{A}$ of the action functional, an arbitrary compact set $K \subset B_{s}$ is defined across the discontinuity $S_{s}$ and the variation of the action functional is performed assuming that the test functions $\delta \chi_{s}$ and $\delta \phi_{s}$ have support included in the set $K$. This means that $\delta \chi_{s}$ and $\delta \phi_{s}$ are assumed to be suitably regular and to vanish on the boundary and outside the set $K$. In the sequel, the intersection of the surface $S_{s}$ with the set $K$ is denoted by $\Sigma$.

Recalling that we assumed $\Psi$ to be a function of $\varepsilon, m_{f}$ and $\chi_{s}$ and that $\Lambda=1 / 2\left(\eta_{s}\left(\mathbf{v}_{s}\right)^{2}+m_{f}\left(\mathbf{v}_{f}^{(s)}\right)^{2}\right)$ it is easy to recognize that

$$
\delta \Psi=\frac{\partial \Psi}{\partial \varepsilon} \mid \delta \varepsilon+\frac{\partial \Psi}{\partial \chi_{s}} \delta \chi_{s}+\frac{\partial \Psi}{\partial m_{f}} \delta m_{f}
$$

and

$$
\delta \Lambda=\eta_{s} \mathbf{v}_{s} \cdot \delta \mathbf{v}_{s}+m_{f} \mathbf{v}_{f}^{(s)} \cdot \delta \mathbf{v}_{f}^{(s)}+\frac{1}{2} \mathbf{v}_{s}^{2} \delta \eta_{s}+\frac{1}{2}\left(\mathbf{v}_{f}^{(s)}\right)^{2} \delta m_{f} .
$$

Recalling Eqs. (82), (83) and (84) and the fact that $\partial \Psi / \partial \varepsilon$ is a symmetric tensor, these equations can be rewritten as

$$
\delta \Psi=\frac{\partial \Psi}{\partial \varepsilon} \mid\left(\mathbf{F}_{s}^{T} \cdot \nabla\left(\delta \chi_{s}\right)\right)+\frac{\partial \Psi}{\partial \chi_{s}} \cdot \delta \chi_{s}+\frac{\partial \Psi}{\partial m_{f}} \operatorname{div}\left(m_{f} \mathbf{G}_{s}^{-1} \cdot \delta \phi_{s}\right)
$$

and

$$
\delta \Lambda=\delta \Lambda_{s}+\delta \Lambda_{f}
$$

where

$$
\begin{aligned}
& \delta \Lambda_{s}:=\left(\eta_{s} \mathbf{v}_{s}+m_{f} \mathbf{v}_{f}^{(s)}\right) \cdot \delta \dot{\chi}_{s}-m_{f} \mathbf{v}_{f}^{(s)} \cdot \nabla\left(\delta \chi_{s}\right) \cdot \mathbf{G}_{s}^{-1} \cdot \mathbf{u}_{s}, \\
& \delta \Lambda_{f}:=\frac{1}{2}\left(\mathbf{v}_{f}^{(s)}\right)^{2} \operatorname{div}\left(m_{f} \mathbf{G}_{s}^{-1} \cdot \delta \phi_{s}\right) \\
& \left.+m_{f} \mathbf{v}_{f}^{(}\right) \mathbf{F}_{s} \cdot \mathbf{G}_{s}^{-1} \cdot \nabla\left(\delta \phi_{s}\right) \cdot \mathbf{G}_{s}^{-1} \cdot \mathbf{u}_{s}-m_{f} \mathbf{v}_{f}^{()} \cdot \mathbf{F}_{s} \cdot \mathbf{G}_{s}^{-1} \cdot \delta \dot{\phi}_{s} .
\end{aligned}
$$

Some processes of integration by parts in space and in time are now needed in order to rewrite the variations $\delta \Psi$ and $\delta \Lambda$ in terms of the variations $\delta \chi_{s}$ and $\delta \phi_{s}$ of the primitive kinematical fields. 
We first compute the variation of the potential energy: integrating by parts in space Eq. (87) it is easy to get

$$
\begin{gathered}
\int_{B_{s} \times(0, T)} \delta \Psi=\int_{K \times(0, T)}\left[\frac{\partial \Psi}{\partial \chi_{s}}-\operatorname{div}\left(\mathbf{F}_{s} \cdot \frac{\partial \Psi}{\partial \varepsilon}\right)\right] \cdot \delta \chi_{s} \\
-\int_{K \times(0, T)}\left[m_{f} \mathbf{G}_{s}^{-T} \cdot \nabla\left(\frac{\partial \Psi}{\partial m_{f}}\right)\right] \cdot \delta \phi_{s}+ \\
\int_{\Sigma \times(0, T)}\left[\left|\mathbf{F}_{s} \cdot \frac{\partial \Psi}{\partial \varepsilon} \mathbf{N}_{s}\right|\right] \cdot \delta \chi_{s}+\int_{\Sigma \times(0, T)}\left[\left|m_{f} \mathbf{G}_{s}^{-T} \cdot \frac{\partial \Psi}{\partial m_{f}} \mathbf{N}_{s}\right|\right] \cdot \delta \phi_{s} .
\end{gathered}
$$

In order to obtain simpler calculations, the variation of the kinetic energy is now computed evaluating separately the two terms appearing in Eq. (87). Integrating by parts in space and time the first term, recalling that $\eta_{s}$ is constant in space and time and that $\mathbf{D}=-m_{f} \mathbf{G}_{s}^{-1} \cdot \mathbf{u}_{s}$ it is easy to recognize that $^{7}$

$$
\begin{aligned}
& \int_{B_{s} \times(0, T)} \delta \Lambda_{s} \\
& \left.=-\int_{K \times(0, T)}\left[\eta_{s} \gamma_{s}+m_{f} \dot{\mathbf{v}}_{f}^{(}\right)+\dot{m}_{f} \mathbf{v}_{f}^{(}+\operatorname{div}\left(\mathbf{v}_{f}^{\circledast} \otimes \mathbf{D}\right)\right] \cdot \delta \chi_{s} \\
& +\int_{\Sigma \times(0, T)}\left(\left[\left|\mathbf{v}_{f}^{(} \otimes \mathbf{D}\right|\right] \cdot \mathbf{N}_{s}\right) \cdot \delta \chi_{s} .
\end{aligned}
$$

Finally, using expression (51) for $\dot{\mathbf{v}}_{f}^{(}$, the balance of mass (56) for $\dot{m}_{f}$ and simplifying, it is easy to recognize that

$$
\begin{aligned}
& \int_{B_{s} \times(0, T)} \delta \Lambda_{s}=-\int_{K \times(0, T)}\left(\eta_{s} \gamma_{s}+m_{f} \gamma_{f}^{\Im}\right) \cdot \delta \chi_{s} \\
& +\int_{\Sigma \times(0, T)}\left(\left[\left|\mathbf{v}_{f}^{(} \otimes \mathbf{D}\right|\right] \cdot \mathbf{N}_{s}\right) \cdot \delta \chi_{s} .
\end{aligned}
$$

The second term appearing on the right hand side of Eq. (87) is now evaluated: integrating by parts in space and time and recalling Eqs. (50) and the definition of the mass flux vector $\mathbf{D}$, we get

\footnotetext{
${ }^{7}$ From now on, we assume that $\delta \chi_{s}(\cdot, 0)=\delta \chi_{s}(\cdot, T)$ and $\delta \phi_{s}(\cdot, 0)=\delta \phi_{s}(\cdot, T)$ so that no time boundary terms are present in our calculation.
} 


$$
\begin{aligned}
& \int_{B_{s} \times(0, T)} \delta \Lambda_{f}=\int_{K \times(0, T)}\left[-\frac{1}{2} m_{f} \nabla\left(\left(\mathbf{v}_{f}^{\Im}\right)^{2}\right) \cdot \mathbf{G}_{s}^{-1}\right] \\
& +\int_{K \times(0, T)}\left[\operatorname{div}\left(\mathbf{v}_{f}^{\Im} \cdot \mathbf{F}_{s} \cdot \mathbf{G}_{s}^{-1} \otimes \mathbf{D}\right)+\frac{\partial}{\partial t}\left(m_{f} \mathbf{v}_{f}^{\Im} \cdot \mathbf{F}_{s} \cdot \mathbf{G}_{s}^{-1}\right)\right] \cdot \delta \phi_{s} \\
& +\int_{\Sigma \times(0, T)}\left[\frac{1}{2}\left[\left|m_{f}\left(\mathbf{v}_{f}^{\Im}\right)^{2} \mathbf{G}_{s}^{-T}\right|\right] \cdot \mathbf{N}_{s}\right. \\
& -\left[\left|\mathbf{v}_{f}^{(} \cdot \mathbf{F}_{s} \cdot \mathbf{G}_{s}^{-1} \otimes \mathbf{D}\right|\right] \cdot \mathbf{N}_{s} \cdot \delta \phi_{s} .
\end{aligned}
$$

Using expressions (56), (51) and (52) for $\dot{m}_{f}, \dot{\mathbf{v}}_{f}^{(s)}$ and $\partial\left(\mathbf{F}_{s} \cdot \mathbf{G}_{s}^{-1}\right) / \partial t$ respectively, recalling again the definition of $\mathbf{D}$ and noting that $\nabla\left(\left(\mathbf{v}_{f}^{\Im}\right)^{2}\right)=$ $2 \mathbf{v}_{f}^{(\Im)} \cdot \nabla \mathbf{v}_{f}^{(\S)}$, this equation can be simplified in

$$
\begin{aligned}
& \int_{B_{s} \times(0, T)} \delta \Lambda_{f}=\int_{K \times(0, T)}\left(m_{f} \gamma_{f}^{\Im} \cdot \mathbf{F}_{s} \cdot \mathbf{G}_{s}^{-1}\right) \cdot \delta \phi_{s} \\
& +\int_{\Sigma \times(0, T)}\left(\left[\left|\frac{1}{2} m_{f} \mathbf{G}_{s}^{-T}\left(\mathbf{v}_{f}^{\Im}\right)^{2}-\mathbf{G}_{s}^{-T} \cdot \mathbf{F}_{s}^{T} \cdot \mathbf{v}_{f}^{(} \otimes \mathbf{D}\right|\right] \cdot \mathbf{N}_{s}\right) \cdot \delta \phi_{s} .
\end{aligned}
$$

Merging Eqs. (88), (89) and (90), and recalling that they hold for any $\delta \chi_{s}, \delta \phi_{s}$ with compact support $K$ included in $B_{s}$, expression (66)for the variation of the action functional is finally recovered.

\section{Appendix: Computation of the Rayleigh-Hamilton Dissipation}

We recall that, owing to (60), (61) and (2), (50), the pull back of the dissipation densities reads

$$
\begin{aligned}
& \mathcal{D}^{(ङ)}=\left(\mathbf{F}_{s} \cdot \mathbf{G}_{s}^{-1} \cdot \mathbf{u}_{s}\right) \cdot \mathbf{K}^{(ङ)} \cdot\left(\mathbf{F}_{s} \cdot \mathbf{G}_{s}^{-1} \cdot \mathbf{u}_{s}\right) \\
& +\left(\nabla \mathbf{v}_{f}^{\Im} \cdot \mathbf{F}_{s}^{-1}\right): \mathbf{M}^{\circledR}:\left(\nabla \mathbf{v}_{f}^{\Im} \cdot \mathbf{F}_{s}^{-1}\right) \\
& +\left(\nabla\left(\mathbf{F}_{s} \cdot \mathbf{G}_{s}^{-1} \cdot \mathbf{u}_{s}\right) \cdot \mathbf{F}_{s}^{-1}\right): \mathbf{B}^{\circledR}:\left(\nabla\left(\mathbf{F}_{s} \cdot \mathbf{G}_{s}^{-1} \cdot \mathbf{u}_{s}\right) \cdot \mathbf{F}_{s}^{-1}\right), \\
& \left.\mathcal{D}_{S}^{(\Im)}=\left[\left|\mathbf{v}_{f}^{(}\right|\right] \cdot \mathbf{S}^{(}\right)\left[\left|\mathbf{v}_{f}^{(\Im}\right|\right] .
\end{aligned}
$$

We consider arbitrary test functions $\delta \mathbf{q}=\left(\delta \chi_{s}, \delta \phi_{s}\right)$ with compact support included in $\mathbb{B}_{s}$ We now compute the term appearing on the right hand side 
of Eq.(65); in particular we start by noticing that owing to Eq. (64) we get

$$
\begin{aligned}
& \left.\int_{0}^{T}\left(\frac{\partial \mathcal{R}}{\partial \dot{\mathbf{q}}_{t}} \mid \delta \mathbf{q}_{t}\right) d t=\int_{\mathbb{B}_{s} \backslash \mathbb{S}_{s}} J_{s}\left(\mathbf{F}_{s} \cdot \mathbf{G}_{s}^{-1} \cdot \delta \phi_{s}\right) \cdot \kappa^{\Im}\right)+ \\
& \int_{\mathbb{B}_{s} \backslash \mathbb{S}_{s}} J_{s}\left(\nabla\left(\delta \chi_{s}-\mathbf{F}_{s} \cdot \mathbf{G}_{s}^{-1} \cdot \delta \phi_{s}\right) \cdot \mathbf{F}_{s}^{-1}\right): \Pi_{f}^{\Im} \\
& +\int_{\mathbb{B}_{s} \backslash \mathbb{S}_{s}} J_{s}\left(\nabla\left(\mathbf{F}_{s} \cdot \mathbf{G}_{s}^{-1} \cdot \delta \phi_{s}\right) \cdot \mathbf{F}_{s}^{-1}\right): \Pi^{\Im}+ \\
& \int_{\mathbb{S}_{s}}\left\|J_{s} \mathbf{F}_{s}^{-T} \cdot \mathbf{N}_{s}\right\|\left[\left|\delta \chi_{s}-\mathbf{F}_{s} \cdot \mathbf{G}_{s}^{-1} \cdot \delta \phi_{s}\right|\right] \cdot \sigma^{\Im} .
\end{aligned}
$$

Integrating by parts in space the right hand side of equation (93) gives

$$
\begin{aligned}
& \int_{\mathbb{B}_{s} \backslash \mathbb{S}_{s}}-\operatorname{div}\left(J_{s} \Pi_{f}^{(\Im)} \cdot \mathbf{F}_{s}^{-T}\right) \cdot \delta \chi_{s} \\
& +\int_{\mathbb{B}_{s} \backslash \mathbb{S}_{s}} \mathbf{G}_{s}^{-T} \cdot \mathbf{F}_{s}^{T} \cdot\left(J_{s} \kappa^{(\Im)}-\operatorname{div}\left(J_{s}\left(\Pi^{\Im}-\Pi_{f}^{\Im}\right)^{T} \cdot \mathbf{F}_{s}^{-T}\right)\right) \cdot \delta \phi_{s} \\
& +\int_{\mathbb{S}_{s}}\left[\left|J_{s} \Pi_{f}^{(s)} \cdot \mathbf{F}_{s}^{-T} \cdot \mathbf{N}_{s}\right|\right] \cdot \delta \chi_{s} \\
& +\int_{\mathbb{S}_{s}}\left[\mid \mathbf{G}_{s}^{-T} \cdot \mathbf{F}_{s}^{T} \cdot\left(J_{s}\left(\left(\Pi^{(}\right)-\Pi_{f}^{\Im}\right)^{T}\right) \cdot \mathbf{F}_{s}^{-T} \cdot \mathbf{N}_{s}\right. \\
& -\left\|J_{s} \mathbf{F}_{s}^{-T} \cdot \mathbf{N}_{s}\right\| \sigma^{(\Im)} \cdot \delta \phi_{s} .
\end{aligned}
$$

\section{Bibliography}

Alazmi B., Vafai K.: Analysis of fluid flow and heat transfer interfacial conditions between a porous medium and a fluid layer. Int. J. Heat Mass Transf. 44, 1735-1749 (2001)

Albers B.: Monochromatic surface waves at the interface between poroelastic and fluid half-spaces. Proc. R. Soc. Lond. A 462, 701723 (2006)

Allaire G.: Homogenization of the Stokes flow in a connected porous medium. Asymptotic Anal. 2, 3, 203-222 (1989).

Allaire G.: Homoge ne isation des e quations de Stokes dans un domaine perfore de petits trous re partis pe riodiquement. C. R. Acad. Sci. Paris, Se r. I, 309, 11, 741-746 (1989).

Allaire G.: Homogenization of the Navier-Stokes equations in open sets perforated with tiny holes. Arch. Rational Mech. Anal., 113, 209298 (1991). 
Allaire G.: Homogenization of the Navier-Stokes equations with a slip boundary condition. Comm. Pure Appl. Math., XLIV, 605-641 (1991).

Allaire, G.: Continuity of the Darcys law in the low-volume fraction limit. Ann. Scuola Norm. Sup. Pisa Cl. Sci. (4) 18, 475-499 (1991)

Altay G., Dokmeci M.C.: On the equations governing the motion of an anisotropic poroelastic material. Proc. R. Soc. A 462, 2373-2396 (2006)

Baek S., Srinivasa A.R.: Diffusion of a fluid through an elastic solid undergoing large deformation. Int. J. Non-Linear Mech. 39, 201-218 (2004)

Batra G., Bedford A., Drumheller D.S.: Applications of Hamiltons principle to continua with singular surfaces. Arch. Rational Mech. Anal. 93, 223251 (1986)

Bedford A., Drumheller D.S.: A variational theory of immiscible mixtures. Arch. Rational Mech. Anal. 68, 37-51 (1978)

Bedford A., Drumheller D.S.: A variational theory of porous media. Int. J. Solids Struct. 15, 967-980 (1979) 20 CISM Course C-1006 Udine, July 12-16 2010

Bedford A., Drumheller D.S.: Recent advances: theories of immiscible and structured mixtures. Int. J. Eng. Sci. 21, 863-960 (1983)

Biot M.A.: General theory of three-dimensional consolidation. J. Appl. Phys. 12, 155-164 (1941)

Biot M.A.: General solutions of the equations of elasticity and consolidation for a porous material. J. Appl. Mech. 23, 91-96 (1956)

Biot M.A.: Theory of propagation of elastic waves in fluid-saturated porous solid. J. Acoust. Soc. Am. 28, 168-191 (1956)

Biot M.A., Willis D G.: The elastic coefficients of the theory of consolidation. J. Appl. Mech. 24 594-601 (1957)

Biot M.A.: Mechanics of deformation and acoustic propagation in porous media. J. Appl. Phys. 33, 1482-1498 (1962)

Biot M.A.: Variational principles for acoustic gravity waves. Phys. Fluids 6, 772-778 (1963)

Beavers G.S., Joseph D.D.: Boundary conditions at a naturally permeable wall. J. Fluid Mech. 30, 197- 207 (1967)

Burridge R., Keller J.B.: Poroelasticity equations derived from microstructure. J. Acoust. Soc. Am. 70:4, 1140-1146 (1981)

Casertano L., Oliveri del Castillo A., Quagliariello M.T.: Hydrodynamics and geodynamics in Campi Flegrei Area of Italy. Nature 264, 161-164 (1976)

Caviglia G.,MorroA.:Harmonic waves in thermo-visco-elastic solids.Int. J. Eng. Sci.43, 1323-1336 (2005)

Chandesris M., Jamet D.: Boundary conditions at a planar fluidporous interface for a Poiseuille flow. Int. J. Heat and Mass Transf. 49, 2137- 2150 
(2006).

Chandesris M., Jamet D.: Boundary conditions at a fluidporous interface: an a priori estimation of the stress jump coefficients. Int. J. Heat and Mass Transf. 50, 3422-3436 (2007)

Chateau X., Dormieux L.: Homoge ne isation dun milieu poreux non sature : Lemme de Hill et applica- tions. C.R. Acad. Sci. Paris 320, Se rie IIb, 627-634 (1995)

Chateau X., Dormieux L.: Approche microme canique du comportement dun milieu poreux non sature . C. R. Acad. Sci. Paris 326, Se rie II, 533-538 (1998)

Cieszko M., Kubik J.: Interaction of elastic waves with a fluid-saturated porous solid boundary. J. of Theor. Appl. Mech. 36, 561580 (1998)

Cieszko M. Kubik J.: Derivation of matching conditions at the contact surface between fluid-saturated porous solid and bulk fluid. Transp. Porous Med. 34, 319336 (1999)

Coussy O., Bourbie T.: Propagation des ondes acoustiques dans les milieux poreux sature s. Rev. Inst. Fr. Pet. 39, 47-66 (1984)

Coussy O. Dormieux L. Detournay E.: From mixture theory to Biots approach for porous media. Int. J. Solids Struct. 35, 46194635 (1998)

Coussy O.: Poromechanics, John Wiley Sons, Chichester (2004)

Cowin SC: Bone Mechanics Handbook, Boca Raton, FL: CRC Press (2001)

Debergue P. et al.: Boundary conditions for the weak formulation of the mixed (u,p) poroelasticity prob- lem. J. Acoust. Soc. Am. 106:5, 2383-2390 (1999)

de Boer R.: Highlights in the historical development of the porous media theory: toward a consistent macroscopic theory. Appl. Mech. Rev. 49:4, 201-262 (1996)

de Boer R.: Contemporary progress in porous media theory. Appl. Mech. Rev. 53, 323370 (2000)

de Boer R.: Theoretical poroelasticity a new approach. Chaos Solitons Fractals 25, 861-878 (2005)

de Buhan P., Dormieux L., Chateau X.: A micro-macro approach to the constitutive formulation of large strain poroelasticity. Poromechanics a tribute to Maurice A. Biot. Thimus et al., Rotterdam (1998) 21 CISM Course C-1006 Udine, July 12-16 2010

de Buhan P., Chateau X., Dormieux L.: The constitutive equations of finite strain poroelasticity in the light of a micro-macro approach. Eur. J. Mech. A/Solids. 17, 909-921 (1998)

Cryer C.W.: A comparison of the three dimensional consolidation theories of Biot and Terzaghi. Q. J. Mech. Appl. Math. 16:4, 401-412 (1963) 
de la Cruz V., Hube J., Spanos T.J.T.: Reflection and transmission of seismic waves at the boundaries of porous media. Wave Motion 16, 323-338 (1992)

dellIsola F., Rosa L., Woz niak C.: A micro-structured continuum modelling compacting fluid-saturated grounds: the effects of pore-size scale parameter. Acta Mech. 127, 1:4, 165-182 (1998)

dellIsola, F., Guarascio, M., Hutter, K.: A variational approach for the deformation of a saturated porous solid. A second gradient theory extending Terzaghis effective stress principle. Arch. Appl. Mech. 70, 323- 337 (2000).

dellIsola, F., Sciarra, G., Batra, R.C.: Static Deformations of a Linear Elastic Porous Body filled with an Inviscid Fluid. J. Elasticity 72, 99-120 (2003).

Deresiewicz H.: The effect of boundaries on wave propagation in a liquidfilled porous solid: I. Reflection of plane waves at a free plane boundary (non-dissipative case). Bull. Seismol. Soc. Am. 50, 599607 (1960)

Deresiewicz H.: The effect of boundaries on wave propagation in a liquidfilled porous solid: III. Reflec- tion of plane waves at a free plane boundary (general case) Bull. Seismol. Soc. Am. 52, 595625 (1962)

Deresiewicz H.: A note on Love waves in a homogeneous crust overlying an inhomogeneous substratum. Bull. Seismol. Soc. Am. 52, 639-645 (1962)

Deresiewicz H.: The effect of boundaries on wave propagation in a liquidfilled porous solid: IV. surface in a half-space. Bull. Seismol. Soc. Am. 50, 627-638 (1962)

Deresiewicz H.: On uniqueness in dynamic poroelasticity. Bull. Seismol. Soc. Am. 53, 783-788 (1963)

Deresiewicz H.: The effect of boundaries on wave propagation in a liquidfilled porous solid: V. Trans- mission across a plane interface. Bull. Seismol. Soc. Am. 54, 409-416 (1964)

Deresiewicz H.: The effect of boundaries on wave propagation in a liquidfilled porous solid: VII. Surface waves in a half-space in the presence of a liquid layer. Bull. Seismol. Soc. Am. 54, 425430 (1964)

Dormieux L.,Coussy O., de Buhan P.: Mode lisation me canique dun milieu polyphasique par la me thode des puissances virtuelles. C.R. Acad. Sci. Paris. 313, Se rie IIb, 863-868 (1991)

Dormieux L., Stolz C.: Approche variationelle en poroe lasticite . C.R. Acad. Sci. Paris, 315, Se rie IIb, 407-412 (1992)

Dormieux L., Kondo D. and Ulm F.-J. Microporomechanics Wiley (2006).

Fillunger P.: Erbdaumechanik. Selbst Verlag des Verfassers, Wien (1936).

Gavrilyuk S.L., Gouin H., Perepechko Yu.V.: A variational principle for two-fluid models. C.R. Acad. Sci. Paris, 324, Se rie IIb, 483-490 (1997)

Gavrilyuk S.L., Gouin H., Perepechko Yu.V.: Hyperbolic Models of Ho- 
mogeneous Two-Fluid Mixtures, Meccanica 33, 161-175(1998)

Gavrilyuk S., Perepechko Yu. V.: Variational approach to constructing hyperbolic models of two-velocity media. J. Appl. Mech. Technical Phys. 39:5, 684-698 (1998)

GavrilyukS.L.,GouinH.:A new form of governing equations of fluids arising from Hamiltons principle. Int. J. Eng. Sci. 37, 1495-1520 (1999)

Gavrilyuk S., Saurel R.: Rankine-Hugoniot relations for shocks in heterogeneous mixtures J. Fluid Mech. 575, 495-507 (2007)

Germain P.: Cours de me canique des milieux continus tome 1 the orie ge ne rale, Masson (1973)

Gouin H.: Variational theory of mixtures in continuum mechanics. Eur. J. Mech., B/Fluids 9, 469-491 (1990)

Gouin H., Gavrilyuk S.L.: Hamiltons principle and Rankine-Hugoniot conditions for general motions of mixtures. Meccanica 34, 39-47 (1998) 22 CISM Course C-1006 Udine, July 12-16 2010 Goyeau B. et al.: Momentum transport at a fluidporous interface. Int. J. Heat Mass Transf. 46, 40714081 (2003)

Haber S., Mauri R.: Boundary conditions for Darcys flow through porous media. Int. J. Multiphase Flow 9, 561574 (1983)

Hassanizadeh S. M. Gray W.: Boundary and interface conditions in porous media. Water Resources Res. 25, 17051715 (1989)

Hornung U.: Homogenization and Porous Media. Interdiscip. Appl. Math., vol. 6, Springer, Berlin (1997)

HoulsbyaG.T.,PuzrinA.M.:Rate-dependent plasticity models derived from potential functions. J. Rheol. 46(1), 113-126 (2002)

Jager W., Mikelic A.: On the interface boundary condition of Beavers, Joseph, and Saffman. SIAM J. Appl. Math. 60, 1111-1127 (2000)

Kaasschieter E.F., Frijns A.J.H.: Squeezing a sponge: a three-dimensional solution in poroelasticity Computational Geosciences 7: 49-59, (2003).

Kosin ski W.: Field Singularities and Wave Analysis in Continuum Mechanics. PWN-Polish Scientific Publishers, Warszawa (1986)

Kubik J., Cieszko M.: Analysis of matching conditions at the boundary surface of a fluid-saturated porous solid and a bulk fluid: the use of Lagrange multipliers. Continuum Mech. Thermodyn. 17:4, 351-359 (2005)

Kuznetsov A.V.: Influence of the stress jump condition at the porous medium/clear fluid interface on a flow at porous wall. Int. Comm. Heat Mass Transf. 24, 401-410. (1997)

Le Bars M., Grae Worster M.: Interfacial conditions between a pure fluid and a porous medium: impli- cations for binary alloy solidification. J. Fluid Mech. 550, 149-173 (2006)

Lee C.K.: Flow and deformation in poroelastic media with moderate 
load and weak inertia. Proc. R. Soc. Lond. A 460, 20512087 (2004)

Levy T., Sanchez-Palencia E.: . On boundary conditions for fluid flow in porous media Int. J. Eng. Sci. 13, 923-940 (1975)

Madeo A., dellIsola F., Ianiro N., Sciarra G.: A variational deduction of second gradient poroelasticity II: an application to the Consolidation Problem. J. Mech. Mater. Struct. 3:4, 607-625 (2008)

Mandel J.: Consolidation des sols (e tude mathe matique). Geotechnique 3, 287-299 (1953)

Marle C.M.: On macroscopic equations governing multiphase flow with diffusion and chemical reactions in porous media. Int. J. Eng. Sci. 20:5, $643662(1982)$

Mobbs S.D.: Variational principles for perfect and dissipative fluid flows. Proc. R. Soc. Lond. A 381, 457468 (1982)

Neale G., Nader W.: Practical significance of Brinkmans extension of Darcys law: coupled parallel flows within a channel and a bounding porous medium. Can. J. Chem. Eng. 52, 475-478 (1974)

Ochoa-Tapia J.A., Whitaker S.: Momentum transfer at the boundary between a porous; medium and a homogeneous fluid I. Theoretical development. Int. J. Heat Mass Transf. 38, 2635-2646 (1995)

Ochoa-Tapia J. A., Whitaker S.: Momentum transfer at the boundary between a porous medium and a homogeneous fluid II. Comparison with experiment. Int. J. Heat Mass Transf. 38, 2647-2655 (1995).

Ochoa-Tapia J.A., Whitaker S.: Momentum jump condition at the boundary between a porous medium and a homogeneous fluid: inertial effects. J. Porous Media 1, 201-217 (1998).

Orsi G.,Petrazzuoli S.M.,Wohletz K.:Mechanical and thermo-fluid behaviour during unrest at the Campi Flegrei caldera (Italy) J. Vulcanol. Geotherm. Res. 91, 453-470 (1999)

Pan Y., Horne R.N.: Generalized Macroscopic Models for Fluid Flow in Deformable Porous Media. Theories Transport in Porous Media 45: 1-27, 2001.

Prat M.: On the boundary conditions at the macroscopic level ${ }^{6}$ Transp. Porous Med. 4, 259-280 (1988) 23 CISM Course C-1006 Udine, July 12-16 2010

Poulikakos D., Kazmierczak M.: Forced convection in a duct partially filled with a porous material. J. Heat Transf. 109, 653-662 (1987)

Quiroga-Goode G., Carcione J.M.: Heterogeneous modelling behaviour at an interface in porous media. Comput. Geosciences 1, 109125 (1997)

Rajagopal K.R. et al.: On boundary conditions for a certain class of problems in mixture theory. Int. J. Eng. Sci. 24, 14531463 (1986)

Rajagopal K.R., Tao L.: Mechanics of mixtures. Ser. Adva. Math. 
Appl. Sci. Vol. 35 (1995)

Rasolofosaon N.J.P., Coussy O.: Propagation des ondes acoustiques dans les milieux poreux sature s: effets dinterface I. Rev. Inst. Fr. Pet. 40, 581$594(1985)$

Rasolofosaon N.J.P., Coussy O.: Propagation des ondes acoustiques dans les milieux poreux sature s: effets dinterface II. Rev. Inst. Fr. Pet. 40, $785802(1985)$

Rasolofosaon N.J.P., Coussy O.: Propagation des ondes acoustiques dans les milieux poreux sature s: effets dinterface III. Rev. Inst. Fr. Pet. 41, $91103(1986)$

Saffman P. G.: On the boundary conditions at the surface of a porous medium. Stud. Appl. Math. L, 93-101 (1971)

Sciarra G., dellIsola F., Ianiro N., Madeo A.: A variational deduction of second gradient poroelasticity I: general theory. J. Mech. Mater. Struct. $3: 3,507-526$ (2008)

Seliger R.L., Whitham G.B.: Variational principles in continuum mechanics. Proc. R. Soc. Lond. A 305, 1-25 (1968)

Sharma M.D.: Wave propagation across the boundary between two dissimilar poroelastic solids. J. Sound Vib. 314, 657-671 (2008)

SonnetA.M., Maffettone P.L. and Virga E.G.: Continuum theory for nematic liquid crystals with tensorial order. J. Non-Newtonian Fluid Mech. 119 51-59 (2004)

V. Terzaghi K.: Theoretical soil mechanics. John Wiley Sons, Chichester (1943)

Savare G., Tomarelli F.: Superposition and chain rule for bounded Hessian functions. Adv. Math. 140, 237-281 (1998)

Vafai K., Thiyagaraja R.: Analysis of flow and heat transfer at the interface region of a porous medium. Int. J. Heat Mass Transf. 30, 13911405 (1987)

Vafai K., Kim S.J.: Analysis of surface enhancement by a porous substrate. J. Heat Transf. 112, 700-706 (1990)

Valde s-Paradaa F.J., Goyeaub B., Ochoa-Tapiaa J.A.: Diffusive mass transfer between a microporous medium and a homogeneous fluid: Jump boundary conditions. Chem. Eng. Sci. 61 1692-1704 (2006)

WilmanskiK.:Waves in porous and granular materials. In: Kinetic and Continuum Theories of Granular and Porous Media, 131-185, K. Hutter and K. Wilmanski(eds.), Springer Wien New York (1999)

Wilmanski K.: A few remarks on Biots model and linear acoustics of poroelastic saturated materials. Soil Dyn. Earthq. Eng. 26, 509-536 (2006)

Yang J.: Importance of flow condition on seismic waves at a saturated porous solid boundary. J. Sound Vib. 221:3, 391413 (1999) 